\title{
Cayley graphs on groups with commutator subgroup of order $2 p$ are hamiltonian
}

\author{
Dave Witte Morris * \\ Department of Mathematics and Computer Science, University of Lethbridge, \\ Lethbridge, Alberta, T1K 3M4, Canada
}

Received 19 March 2017, accepted 21 December 2017, published online 17 January 2018

\begin{abstract}
We show that if $G$ is a finite group whose commutator subgroup $[G, G]$ has order $2 p$, where $p$ is an odd prime, then every connected Cayley graph on $G$ has a hamiltonian cycle.

Keywords: Cayley graph, hamiltonian cycle, commutator subgroup.

Math. Subj. Class.: 05C25, 05C45
\end{abstract}

\section{Introduction}

Let $G$ be a finite group. It is easy to show that if $G$ is abelian (and $|G|>2$ ), then every connected Cayley graph on $G$ has a hamiltonian cycle. (See Definition 2.1 for the definition of the term Cayley graph.) To generalize this observation, one can try to prove the same conclusion for groups that are close to being abelian. Since a group is abelian precisely when its commutator subgroup is trivial, it is therefore natural to try to find a hamiltonian cycle when the commutator subgroup of $G$ is close to being trivial. The following theorem, which was proved in a series of papers, is a well-known result along these lines.

Theorem 1.1 (Marušič [13], Durnberger [4, 5], 1983-1985). If the commutator subgroup $[G, G]$ of $G$ has prime order, then every connected Cayley graph on $G$ has a hamiltonian cycle.

D. Marušič (personal communication) suggested more than thirty years ago that it should be possible to replace the prime with a product $p q$ of two distinct primes:

Problem 1.2 (D. Marušič, personal communication, 1985). Show that if the commutator subgroup of $G$ has order $p q$, where $p$ and $q$ are two distinct primes, then every connected Cayley graph on $G$ has a hamiltonian cycle.

\footnotetext{
*Homepage: http://people.uleth.ca/ dave.morris/

E-mail address: Dave.Morris@uleth.ca (Dave Witte Morris)
} 
This has recently been accomplished when $G$ is either nilpotent [8] or of odd order [16]. As another step toward the solution of this problem, we establish the special case where $q=2$ :

Theorem 1.3. If the commutator subgroup of $G$ has order $2 p$, where $p$ is an odd prime, then every connected Cayley graph on $G$ has a hamiltonian cycle.

See the bibliography of [12] for references to other results on hamiltonian cycles in Cayley graphs.

The proof of Theorem 1.3 is a lengthy case-by-case analysis, based on the choice of certain elements $a$ and $b$ of the Cayley graph's connection set (see Notation 3.3). Here is an outline of the paper:

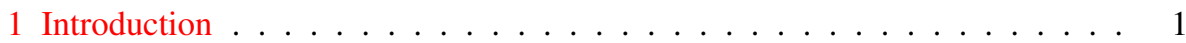

2 Some known results . . . . . . . . . . . . . . . . . . 2

3 Assumptions, group theory, and connected sums . . . . . . . . . . . . 6

4 Case with $\bar{s}=\bar{t} \ldots \ldots \ldots \ldots \ldots \ldots \ldots$

5 Cases with $|\bar{a}|>2$ and $\bar{b} \notin\langle\bar{a}\rangle \ldots \ldots \ldots \ldots . \ldots \ldots \ldots$

6 Cases with $\bar{b} \in\langle\bar{a}\rangle \ldots \ldots \ldots \ldots \ldots$

7 Cases with $|\bar{a}|=2$ and $\# S=2 \ldots \ldots \ldots \ldots$

8 Cases with $|\bar{a}|=2$ and $\# S=3 \ldots \ldots \ldots$. . . . . . . . . . . . . . . . . . . . . . . 23

9 Cases with $|\bar{a}|=2$ and $\# S \geq 4 \ldots \ldots \ldots$

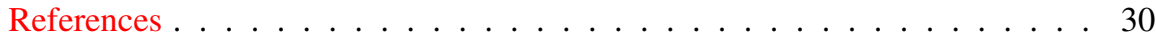

\section{Some known results}

We recall a few results that provide hamiltonian cycles in various Cayley graphs.

Definition 2.1 (cf. [9, p. 34]). For any subset $S$ of a finite group $G$, Cay $(G ; S)$ is the graph whose vertex set is $G$, with an edge joining $g$ to $g s$, for each $g \in G$ and $s \in S$. This is called the Cayley graph of the connection set $S$ on the group $G$.

Remark 2.2. Unlike most authors (including [9]), we do not require the connection set $S$ to be symmetric in the definition of a Cayley graph; that is, we do not assume $S$ is closed under inverses. This does not change the set of graphs that are considered to be Cayley graphs, because, in our notation, $\operatorname{Cay}(G ; S)=\operatorname{Cay}\left(G ; S \cup S^{-1}\right)$, where $S^{-1}=\left\{s^{-1} \mid s \in S\right\}$.

Theorem 2.3 ([3, 6, 7, 12]). Every connected Cayley graph on $G$ has a hamiltonian cycle if $|G|=k p$ for some prime $p$ and some $k \in \mathbb{N}$ with $1 \leq k<32$ and $k \neq 24$.

\section{Notation 2.4.}

- The symbol $G$ always represents a finite group.

- For $g \in G$ and $s_{1}, \ldots, s_{n} \in S \cup S^{-1}$, we use $[g]\left(s_{1}, \ldots, s_{n}\right)$ to denote the walk in $\operatorname{Cay}(G ; S)$ that visits (in order), the vertices

$$
g, g s_{1}, g s_{1} s_{2}, g s_{1} s_{2} s_{3}, \ldots, g s_{1} s_{2} \cdots s_{n} .
$$

We may write $\left(s_{1}, \ldots, s_{n}\right)$ for $[e]\left(s_{1}, \ldots, s_{n}\right)$.

- We use $\left(s_{1}, \ldots, s_{n}\right)^{k}$ to denote the concatenation of $k$ copies of the sequence $\left(s_{i}\right)_{i=1}^{n}$. 
- Appending \# to a sequence deletes the last term; that is, $\left(s_{i}\right)_{i=1}^{n} \#=\left(s_{i}\right)_{i=1}^{n-1}$.

- If $W=[g]\left(s_{1}, \ldots, s_{n}\right)$ is a walk in $\operatorname{Cay}(G ; S)$, and $h \in G$, we use $h W$ to denote the translate $[h g]\left(s_{1}, \ldots, s_{n}\right)$.

- When $C$ is an oriented cycle, we use $-C$ to denote the same cycle as $C$, but with the opposite orientation.

- For $g, h \in G$ :

$$
[g, h]=g^{-1} h^{-1} g h, \quad g^{h}=h^{-1} g h, \quad \text { and } \quad{ }^{h} g=h g h^{-1}\left(=g^{h^{-1}}\right) .
$$

- We use $G^{\prime}$ to denote the commutator subgroup $[G, G]$ of $G$.

- For convenience, we let $\bar{G}=G / G^{\prime}$.

- For $g \in G$, we let $\bar{g}=g G^{\prime}$ be the image of $g$ in $\bar{G}$.

- We use $Z(G)$ to denote the center of $G$.

Definition 2.5 (cf. [10, $§ 2.1 .3$, p. 61]). Suppose

- $N$ is an abelian, normal subgroup of $G$, and

- $C=[N v]\left(s_{i}\right)_{i=1}^{n}$ is an (oriented) cycle in $\operatorname{Cay}(G / N ; S)$.

The voltage of $C$ is ${ }^{v}\left(\prod_{i=1}^{n} s_{i}\right)$. This is an element of $N$, and it may be denoted $\Pi C$.

We have the following straightforward observations:

Lemma 2.6. Assume the notation of Definition 2.5. Then:

1. $\Pi C$ is determined by the oriented cycle $C$ : it is independent of the choice of the vertex $N v$ of $C$, and of the choice of the representative $v$ of $N v$.

2. $\Pi g C={ }^{g}(\Pi C)$ for all $g \in G$.

3. $\Pi(-C)=(\Pi C)^{-1}$.

Definition 2.7. A subset $S$ of $G$ is an irredundant generating set of $G$ if $S$ generates $G$, but no proper subset of $S$ generates $G$.

Lemma 2.8 ("Factor Group Lemma” [15, §2.2]). Suppose

- $N$ is a cyclic, normal subgroup of $G$,

- $\left(s_{i}\right)_{i=1}^{m}$ is a hamiltonian cycle in $\operatorname{Cay}(G / N ; S)$, and

- the voltage $\Pi\left(s_{i}\right)_{i=1}^{m}$ generates $N$.

Then $\left(s_{1}, s_{2}, \ldots, s_{m}\right)^{|N|}$ is a hamiltonian cycle in $\operatorname{Cay}(G ; S)$.

Corollary 2.9 ([12, Cor. 2.11]). Suppose

- $N$ is a normal subgroup of $G$, such that $|N|$ is prime,

- the image of $S$ in $G / N$ is an irredundant generating set of $G / N$,

- there is a hamiltonian cycle in $\operatorname{Cay}(G / N ; S)$, and

- $s \equiv t(\bmod N)$ for some $s, t \in S \cup S^{-1}$ with $s \neq t$. 
Then there is a hamiltonian cycle in $\operatorname{Cay}(G ; S)$.

Lemma 2.10 ([2, Lem. 1 on p. 24]). Let $P_{k} \square P_{\ell}$ be the Cartesian product of a path of length $k$ with a path of length $\ell$. If $k \ell$ is even, and $k, \ell \geq 2$, then $P_{k} \square P_{\ell}$ has a hamiltonian path from any corner vertex $v$ to any vertex that is at odd distance from $v$.

Corollary 2.11. Suppose $N$ is a subgroup of an abelian group $H$, and $\{x, y\} \cup S_{0}$ is a subset of $H$ that generates $H / N$. Let $k=|\langle x, N\rangle: N|$ and $\ell=|\langle x, y, N\rangle:\langle x, N\rangle|$. If $k \ell$ is even, $k, \ell \geq 2,0 \leq p<k, 0 \leq q<\ell$, and $p+q$ is odd, then $\operatorname{Cay}\left(H / N ;\{x, y\} \cup S_{0}\right)$ has a hamiltonian path $\left(s_{i}\right)_{i=1}^{r}$, such that $s_{1} s_{2} \cdots s_{r}=x^{p} y^{q}$.

Proof. If we identify the vertices of $P_{k} \square P_{\ell}$ with $\{(i, j) \mid 0 \leq i<k, 0 \leq j<\ell\}$ in the natural way, then the map $(i, j) \mapsto x^{i} y^{j}$ is an isomorphism from $P_{k} \square P_{\ell}$ to a subgraph $X$ of Cay $(\langle x, y\rangle ; x, y)$. So Lemma 2.10 provides a hamiltonian path $\left(t_{i}\right)_{i=1}^{k \ell-1}$ in $X$ from $e$ to $x^{p} y^{q}$. So $t_{1} t_{2} \cdots t_{k \ell-1}=x^{p} y^{q}$.

Let $L=\left(u_{j}\right)_{j=1}^{n}$ be a hamiltonian path in Cay $(H /\langle x, y, N\rangle)$, and let

$$
\left(s_{i}\right)_{i=1}^{r}=\left(L, t_{2 i-1}, L^{-1}, t_{2 i}\right)_{i=1}^{k \ell / 2} \# .
$$

From the definition of $k$ and $\ell$, we see that the natural map from $X$ to the Cayley graph Cay $(\langle x, y, N\rangle / N ; x, y)$ is an isomorphism onto a spanning subgraph. Therefore, $\left(s_{i}\right)_{i=1}^{r}$ is a hamiltonian path in $\operatorname{Cay}(H / N ; S)$. Since $H$ is abelian, it is easy to see that $s_{1} s_{2} \cdots s_{r}=$ $x^{p} y^{q}$.

Given a hamiltonian cycle $C_{0}$ in $\operatorname{Cay}(\bar{G} ; S)$, the following result often provides a second hamiltonian cycle $C_{1}$, such that the voltage of at least one of these two cycles generates $G^{\prime}$. (Then the Factor Group Lemma (2.8) provides a hamiltonian cycle in $\operatorname{Cay}(G ; S)$.)

Lemma 2.12 (cf. Marušič [13] and Durnberger [4], or see [16, Lem. 3.1]). Assume:

- $N$ is an abelian normal subgroup of $G$, such that $G / N$ is abelian,

- $C_{0}$ is an oriented hamiltonian cycle in $\operatorname{Cay}(G / N ; S)$,

- $s, t, u \in S^{ \pm 1}$ and $h \in G$,

- $C_{0}$ contains:

$\circ$ the oriented path $\left[\overline{h s^{-1} u^{-1}}\right]\left(s, t, s^{-1}\right)$, and

$\circ$ either the oriented edge $[\bar{h}](t)$ or the oriented edge $[\overline{h t}]\left(t^{-1}\right)$.

Then there is a hamiltonian cycle $C_{1}$ in $\operatorname{Cay}(G / N ; S)$, such that

$$
\left(\left(\Pi C_{0}\right)^{-1}\left(\Pi C_{1}\right)\right)^{h}= \begin{cases}{\left[u, t^{-1}\right]\left[s, t^{-1}\right]^{u}} & \text { if } C_{0} \text { contains }[\bar{h}](t), \\ {\left[t^{-1}, u\right]\left[s, t^{-1}\right]^{u}} & \text { if } C_{0} \text { contains }[\overline{h t}]\left(t^{-1}\right) .\end{cases}
$$

Furthermore, $C_{0}$ and $C_{1}$ have exactly the same oriented edges, except for some of the edges in the subgraph induced by $\left\{\bar{h}, \overline{h u^{-1}}, \overline{h s^{-1} u^{-1}}, \overline{h t}, \overline{h t u^{-1}}, \overline{h t s^{-1} u^{-1}}\right\}$.

Lemma 2.13 ([4, Lem. 2.8]). Assume

- $S$ is an irredundant generating set of $G$,

- $s, t \in S$, with $s \neq t$, 
- $s$ commutes with $t$,

- $\langle S \backslash\{s\}\rangle \triangleleft G$, and

- there is a hamiltonian cycle in $\operatorname{Cay}(\langle S \backslash\{s\}\rangle ; S \backslash\{s\})$.

Then there is a hamiltonian cycle in $\operatorname{Cay}(G ; S)$.

We do not need the general theory of nilpotent groups, but we will make use of the following two facts. (The first is essentially the definition of a nilpotent group, which can be found in any graduate-level textbook on group theory.)

Lemma 2.14 ([14, (iii) on p. 175 and Prop. VI.1.h on page 176]).

1. Every abelian group is nilpotent.

2. If $G / Z(G)$ is nilpotent, then $G$ is nilpotent.

Therefore, if $G^{\prime} \subseteq Z(G)$ (in other words, if $G / Z(G)$ is abelian), then $G$ is nilpotent.

Theorem 2.15 ([8]). If $G$ is a nontrivial, nilpotent, finite group, and the commutator subgroup of $G$ is cyclic, then every connected Cayley graph on $G$ has a hamiltonian cycle.

The following observation is well known (and easy to prove).

Lemma 2.16 ([12, Lem. 2.27]). Let $S$ generate a finite group $G$ and let $s \in S$, such that $\langle s\rangle \triangleleft G$. If

- Cay $(G /\langle s\rangle ; S)$ has a hamiltonian cycle, and

- either

1. $s \in Z(G)$, or

2. $Z(G) \cap\langle s\rangle=\{e\}$, or

3. $|s|$ is prime,

then $\operatorname{Cay}(G ; S)$ has a hamiltonian cycle.

\section{Corollary 2.17. Suppose}

- $G^{\prime}$ is cyclic of order $p q$, where $p$ and $q$ are distinct primes,

- $S$ is an irredundant generating set of $G$, and

- some nontrivial element s of $S$ is in $G^{\prime}$.

Then $\operatorname{Cay}(G ; S)$ has a hamiltonian cycle.

Proof. We may assume $G^{\prime}=\mathbb{Z}_{p} \times \mathbb{Z}_{q}$. Since every subgroup of a cyclic, normal subgroup is also normal, we know that $\langle s\rangle \triangleleft G$. Also, there are hamiltonian cycles in $\operatorname{Cay}\left(G / \mathbb{Z}_{p} ; S\right)$, $\operatorname{Cay}\left(G / \mathbb{Z}_{q} ; S\right)$, and $\operatorname{Cay}\left(G / G^{\prime} ; S\right)$ (by Theorem 1.1 and the elementary fact that Cayley graphs on abelian groups have hamiltonian cycles). Hence, we may assume $\langle s\rangle=G^{\prime}$ and $G^{\prime} \cap Z(G)=\mathbb{Z}_{q}$ (perhaps after interchanging $p$ and $q$ ), for otherwise Lemma 2.16 applies.

Let $\widehat{G}=G / \mathbb{Z}_{p}$. We may assume $|\widehat{G}| \neq 27$, for otherwise $|G|=27 p$ so Theorem 2.3 applies. Then, since $\widehat{G}$ is nilpotent (see Lemma 2.14) and its commutator subgroup is $\mathbb{Z}_{q}$, the proof in $[11, \S 4]$ implies there is a hamiltonian cycle $\left(t_{i}\right)_{i=1}^{n}$ in $\operatorname{Cay}\left(\widehat{G} / \widehat{G}^{\prime} ; S^{\prime}\right)$ whose 
voltage generates $\widehat{G}^{\prime}$. Then, since $\mathbb{Z}_{p} \cap Z(G)=\{e\}$, the proof of Lemma 2.16(2) in [12, Lem. 2.27(2)] tells us that $\left(t_{i}, s^{p-1}\right)_{i=1}^{n}$ is a hamiltonian cycle in $\operatorname{Cay}\left(G / \mathbb{Z}_{q} ; S\right)$.

Note that, since $\widehat{G}$ is a nilpotent group whose commutator subgroup is in the center and has prime order $q$, the order of $\left|\widehat{G} / G^{\prime}\right|$ must be a multiple of $q$; that is, $n$ is a multiple of $q$ (cf. Lemma 3.6 below). Calculating modulo $\mathbb{Z}_{p}$, we have

$$
\begin{aligned}
\Pi\left(t_{i}, s^{p-1}\right)_{i=1}^{n} & \equiv s^{(p-1) n} \Pi\left(t_{i}\right)_{i=1}^{n} & & \left(\widehat{s} \in \widehat{G}^{\prime}=\widehat{\mathbb{Z}_{q}} \subseteq Z(\widehat{G})\right) \\
& \equiv \Pi\left(t_{i}\right)_{i=1}^{n} & & (n \text { is a multiple of } q) \\
& \neq \equiv & & \left(\Pi\left(t_{i}\right)_{i=1}^{n} \text { generates } \widehat{G}^{\prime}\right) .
\end{aligned}
$$

Therefore $\Pi\left(t_{i}, s^{p-1}\right)_{i=1}^{n}$ generates $\mathbb{Z}_{q}$. So the Factor Group Lemma (2.8) tells us that $\left(\left(t_{i}, s^{p-1}\right)_{i=1}^{n}\right)^{q}$ is a hamiltonian cycle in $\operatorname{Cay}(G ; S)$.

\section{Assumptions, group theory, and connected sums}

Assumptions 3.1. The remainder of this paper provides a proof of Theorem 1.3, so

- $p$ is an odd prime,

- $G$ is a finite group whose commutator subgroup has order $2 p$, and

- $S$ is an irredundant generating set of $G$.

We wish to show that the Cayley graph $\operatorname{Cay}(G ; S)$ has a hamiltonian cycle.

\section{A Basic group theory}

Assumption 3.2. Because of Corollary 2.17, we may assume $S \cap G^{\prime}=\emptyset$.

Notation 3.3. The assumption that the commutator subgroup has order $2 p$ implies that $G^{\prime}$ is cyclic (cf. [16, $\S 2 \mathrm{E}$, proof of Cor. 1.4]), so we may write

$$
G^{\prime}=\mathbb{Z}_{2} \times \mathbb{Z}_{p}
$$

From Theorem 2.15, we may assume that $G$ is not nilpotent, so $G^{\prime} \nsubseteq Z(G)$ (see Lemma 2.14). This implies $\mathbb{Z}_{p} \cap Z(G)=\{e\}$. Hence there exists $a \in S$, such that

$$
a \text { does not centralize } \mathbb{Z}_{p}
$$

Then there exists $b \in S$, such that

$$
\mathbb{Z}_{p} \subseteq\langle[a, b]\rangle
$$

The assumptions (3.3A) and (3.3B) are the basis of most of the arguments in the later sections of the paper.

For ease of reference, we now collect a few well-known facts from group theory (specialized to our setting).

Lemma 3.4. If $S_{0} \subseteq G$, such that $\left\langle S_{0}, \mathbb{Z}_{2}\right\rangle=G$, then $\left\langle S_{0}\right\rangle=G$. 
Proof. Since $\mathbb{Z}_{2} \subseteq Z(G)$, we have

$$
\left\langle S_{0}\right\rangle^{\prime}=\left\langle S_{0}, Z(G)\right\rangle^{\prime} \supseteq\left\langle S_{0}, \mathbb{Z}_{2}\right\rangle^{\prime}=G^{\prime} .
$$

Therefore

$$
\left\langle S_{0}\right\rangle=\left\langle S_{0},\left\langle S_{0}\right\rangle^{\prime}\right\rangle=\left\langle S_{0}, G^{\prime}\right\rangle \supseteq\left\langle S_{0}, \mathbb{Z}_{2}\right\rangle=G
$$

Corollary 3.5. Suppose $S_{0}$ is a proper subset of $S$, such that $\mathbb{Z}_{p} \subseteq\left\langle S_{0}\right\rangle$. (In particular, this will be the case if $\{a, b\} \subseteq S_{0}$.) Then $\left\langle\overline{S_{0}}\right\rangle \neq \bar{G}$.

Proof. Suppose $\left\langle\overline{S_{0}}\right\rangle=\bar{G}$. This means $\left\langle S_{0}, G^{\prime}\right\rangle=G$. Since $G^{\prime}=\mathbb{Z}_{2} \times \mathbb{Z}_{p}$ and $\mathbb{Z}_{p} \subseteq\left\langle S_{0}\right\rangle$, this implies $\left\langle S_{0}, \mathbb{Z}_{2}\right\rangle=G$. So Lemma 3.4 tells us that $\left\langle S_{0}\right\rangle=G$. This contradicts the fact that the generating set $S$ is irredundant.

Lemma 3.6. Let $H$ be a group. If $x, y, z \in H$, and $y$ centralizes $H^{\prime}$, then $[x y, z]=$ $[x, z][y, z]$. Therefore $\left[y^{k}, z\right]=[y, z]^{k}$ for all $k \in \mathbb{Z}$.

Corollary 3.7. If $x, y \in G$, such that $y$ centralizes $G^{\prime}$, and $\mathbb{Z}_{p} \subseteq\langle[x, y]\rangle$, then $|y|$ is divisible by $p$.

Corollary 3.8. Let $S_{0} \subseteq G$, such that $\mathbb{Z}_{2} \nsubseteq\left\langle S_{0}\right\rangle^{\prime}$. If $g \in G$, such that $\mathbb{Z}_{2} \subseteq\left\langle g, S_{0}\right\rangle^{\prime}$, then $\left|\left\langle\bar{g}, \overline{S_{0}}\right\rangle:\left\langle\overline{S_{0}}\right\rangle\right|$ is even.

In particular, if $\mathbb{Z}_{2} \subseteq\langle[g, h]\rangle$, then, by taking $S_{0}=\{h\}$, we see that $|\langle\bar{g}, \bar{h}\rangle:\langle\bar{h}\rangle|$ is even, so $|\bar{g}|$ is even (and, similarly, $|\bar{h}|$ must also be even).

Corollary 3.9. $|\bar{G}|$ is divisible by 4 .

\section{B Connected sums}

Definition 3.10 ([8, Defn. 5.1]). Assume $C_{1}$ and $C_{2}$ are two vertex-disjoint oriented cycles in $\operatorname{Cay}(\bar{G} ; S)$, and let $g \in G$, and $s, t \in S \cup S^{-1}$. If

- $C_{1}$ contains the oriented edge $[\bar{g}](t)$, and

- $C_{2}$ contains the oriented edge $[\overline{g s t}]\left(t^{-1}\right)$,

then we use $C_{1} \#_{t}^{s} C_{2}$ to denote the oriented cycle obtained from $C_{1} \cup C_{2}$ by

- removing the oriented edges $[\bar{g}](t)$ and $\left[\overline{g s t}\left(t^{-1}\right)\right.$, and

- inserting the oriented edges $[\bar{g}](s)$ and $[\overline{g s t}]\left(s^{-1}\right)$.

This is called the connected sum of $C_{1}$ and $C_{2}$.

If $[g](t)$ is any oriented edge of an oriented cycle $C$, and $s \in S$, such that $s C$ is vertex disjoint from $C$, then we can form the connected sum $C \#_{t}^{s}-s C$. This construction can be iterated:

Definition 3.11. Suppose

- $\left[g_{1}\right]\left(t_{1}\right), \ldots,\left[g_{k}\right]\left(t_{k}\right)$ are oriented edges of an oriented cycle $C$ in $\operatorname{Cay}(\bar{G} ; S)$, such that $g_{i} \neq g_{i+1}$ for all $i$, and 
- $s_{1}, s_{2}, \ldots, s_{k} \in S \cup S^{-1}$, such that the cycles $C, s_{1} C, s_{2} s_{1} C, \ldots, s_{k} s_{k-1} \cdots s_{1} C$ are pairwise vertex-disjoint.

Then we can form the connected sum

$$
C \#_{t_{1}}^{s_{1}}-s_{1} C \#_{t_{2}}^{s_{2}} s_{2} s_{1} C \#_{t_{3}}^{s_{3}} \cdots \#_{t_{k}}^{s_{k}} \pm s_{k} s_{k-1} \cdots s_{1} C .
$$

We call this a connected sum of signed translates of $C$.

Lemma 3.12 (cf. [8, Lem. 5.2]). If $C_{1}, C_{2}, g, s$, and t are as in Definition 3.10, then

$$
\Pi\left(C_{1} \#_{t}^{s} C_{2}\right)=\Pi C_{1} \cdot{ }^{g}\left[s^{-1}, t^{-1}\right] \cdot \Pi C_{2} .
$$

Proof. We may assume $g=t^{-1}$ (or, in other words, $g t=e$ ), after translating the cycles by $(g t)^{-1}$ (cf. Lemma 2.6(2)). Write $C_{1}=\left(s_{i}\right)_{i=1}^{m}$ and $C_{2}=\left[s t^{-1}\right]\left(t_{j}\right)_{j=1}^{n}$, so

$$
\left(C_{1} \#_{t}^{s} C_{2}\right)=\left(\left(s_{i}\right)_{i=1}^{m-1}, s,\left(t_{j}\right)_{j=1}^{n-1}, s^{-1}\right) .
$$

By assumption, $C_{1}$ contains the edge $\overline{t^{-1}} \rightarrow \bar{e}$ and $C_{2}$ contains the edge $\bar{s} \rightarrow \overline{s t^{-1}}$, so $s_{m}=t$ and $t_{n}=t^{-1}$. Therefore

$$
\begin{aligned}
\Pi\left(C_{1} \#_{t}^{s} C_{2}\right) & =\prod_{i=1}^{m-1}\left(s_{i}\right) \cdot s \cdot \prod_{j=1}^{n-1}\left(t_{j}\right) \cdot s^{-1} \\
& =\prod_{i=1}^{m}\left(s_{i}\right) \cdot t^{-1} s \cdot \prod_{j=1}^{n}\left(t_{j}\right) \cdot t s^{-1} \\
& =\Pi C_{1} \cdot t^{-1} s \cdot\left(\Pi C_{2}\right)^{s t^{-1}} \cdot t s^{-1} \\
& =\Pi C_{1} \cdot t^{-1} s t s^{-1} \cdot \Pi C_{2} \\
& =\Pi C_{1} \cdot{ }^{-1}\left[s^{-1}, t^{-1}\right] \cdot \Pi C_{2} \\
& =\Pi C_{1} \cdot g\left[s^{-1}, t^{-1}\right] \cdot \Pi C_{2} .
\end{aligned}
$$

Corollary 3.13. Assume that $C_{1}, C_{2}, g, s$, and $t$ are as in Definition 3.10. If $C_{0}$ is another oriented cycle that is vertex-disjoint from $C_{2}$ and contains the oriented edge $\bar{g}(t)$, then

$$
\left(\Pi\left(C_{0} \#_{t}^{s} C_{2}\right)\right)\left(\Pi\left(C_{1} \#_{t}^{s} C_{2}\right)\right)^{-1}=\left(\Pi C_{0}\right)\left(\Pi C_{1}\right)^{-1} .
$$

Corollary 3.14 ([8, Lem. 5.2]). If $C_{1}, C_{2}, g, s$, and $t$ are as in Definition 3.10, then

$$
\Pi\left(C_{1} \#_{t}^{s} C_{2}\right) \equiv \Pi C_{1} \cdot \Pi C_{2} \cdot[s, t] \quad\left(\bmod \mathbb{Z}_{p}\right) .
$$

The following result describes a fairly common situation in which the connected sum provides hamiltonian cycles in $\operatorname{Cay}(G ; S)$ :

Lemma 3.15. Let $S_{0}$ be a nonempty subset of $S, g \in G, c \in S \backslash S_{0}$, and $s, t \in S \backslash\{c\}$. Assume $C_{0}$ and $C_{1}$ are oriented hamiltonian cycles in Cay $\left(\left\langle\overline{S_{0}}\right\rangle ; S_{0}\right)$, such that

- $\left(\Pi C_{0}\right)^{-1}\left(\Pi C_{1}\right)$ is a nontrivial element of $\mathbb{Z}_{p}$,

- $C_{0}$ and $C_{1}$ both contain the oriented edge $[\bar{g}](s)$, 
- for every $x \in S_{0}, C_{0}$ contains at least two edges that are labelled either $x$ or $x^{-1}$,

- $\mathbb{Z}_{2} \subseteq\langle[c, t]\rangle$, and

- either $\left|\bar{G}:\left\langle\overline{S_{0}}\right\rangle\right|>2$ or $s=t$.

\section{If either}

1. there exists $u \in S \backslash\{c\}$, such that $\mathbb{Z}_{2} \nsubseteq\langle[u, c]\rangle$, or

2. $\left|\bar{G}:\left\langle\overline{S_{0}}, \bar{t}\right\rangle\right|$ is even,

then there is a hamiltonian cycle $C$ in $\operatorname{Cay}(\bar{G} ; S)$, such that $\langle\Pi C\rangle=G^{\prime}$, so the Factor Group Lemma (2.8) yields a hamiltonian cycle in $\operatorname{Cay}(G ; S)$.

Proof. Let $r=\left|\bar{G}:\left\langle\overline{S_{0}}\right\rangle\right|$. We have $\mathbb{Z}_{p} \subseteq\left\langle\left(\Pi C_{0}\right)^{-1}\left(\Pi C_{1}\right)\right\rangle \subseteq\left\langle S_{0}\right\rangle$, so Corollary 3.5 implies $r \neq 1$.

Suppose $r=2$. By assumption, this implies $s=t$, which means that $C_{0}$ and $C_{1}$ both contain the oriented edge $[\bar{g}](t)$. Then the translate $c C_{0}$ contains the oriented edge $[\overline{g c}](t)$. The connected sums $C=C_{0} \#_{t}^{c}-c C_{0}$ and $C^{\prime}=C_{1} \#_{t}^{c}-c C_{0}$ are hamiltonian cycles in $\operatorname{Cay}(\bar{G} ; S)$. From Corollary 3.14, we have

$$
\Pi C \equiv \Pi C_{0} \cdot \Pi C_{0} \cdot[c, t] \equiv[c, t] \not \equiv 0 \quad\left(\bmod \mathbb{Z}_{p}\right)
$$

so $\Pi C$ projects nontrivially to $\mathbb{Z}_{2}$. Corollary 3.13 says $(\Pi C)\left(\Pi C^{\prime}\right)^{-1}=\left(\Pi C_{0}\right)\left(\Pi C_{1}\right)^{-1}$, which generates $\mathbb{Z}_{p}$ (because it is conjugate to the inverse of $\left(\Pi C_{0}\right)^{-1}\left(\Pi C_{1}\right)$, which is assumed to be a nontrivial element of $\mathbb{Z}_{p}$ ). Therefore, we see that either $\Pi C$ or $\Pi C^{\prime}$ generates $G^{\prime}$, as desired. So we may assume henceforth that $r>2$.

We now show that we may assume $t \in S_{0}$. To this end, suppose it is not the case that $t \in S_{0}$. Let $n=\left|\left\langle\overline{S_{0}}, \bar{t}\right\rangle:\left\langle\overline{S_{0}}\right\rangle\right|$. Then, by choosing a sequence $\left\{\left[g_{i}\right]\left(s_{i}\right)\right\}_{i=1}^{n-1}$ of oriented edges of $C_{0}$, we can form a connected sum $C_{0}^{\prime}$ of signed translates of $C_{0}$ :

$$
C_{0}^{\prime}=C_{0} \#_{s_{1}}^{t}-t C_{0} \#_{s_{2}}^{t} \cdots \#_{s_{n-1}}^{t} \pm t^{n-1} C_{0} .
$$

This is a hamiltonian cycle in $\operatorname{Cay}\left(\left\langle\overline{S_{0}}, \bar{t}\right\rangle ; S_{0} \cup\{t\}\right)$. We may assume $s_{1}=s$. Then another hamiltonian cycle $C_{1}^{\prime}$ can be constructed by replacing the leftmost occurrence of $C_{0}$ with $C_{1}$, and Lemma 3.12 tells us that $\left(\Pi C_{0}^{\prime}\right)\left(\Pi C_{1}^{\prime}\right)^{-1}=\left(\Pi C_{0}\right)\left(\Pi C_{1}\right)^{-1}$, which is a nontrivial element of $\mathbb{Z}_{p}$ (and $\left(\Pi C_{0}\right)^{-1}\left(\Pi C_{1}\right)$ is conjugate to the inverse of this). From the definition of connected sum, it is obvious that $C_{0}^{\prime}$ contains at least two edges labelled $t^{ \pm 1}$. So the hamiltonian cycles $C_{0}^{\prime}$ and $C_{1}^{\prime}$ satisfy the hypotheses of the lemma with $S_{0} \cup\{t\}$ in the role of $S_{0}$ and with $t$ in the role of $s$.

Case 1. Assume there exists $u \in S \backslash\{c\}$, such that $\mathbb{Z}_{2} \nsubseteq\langle[u, c]\rangle$.

Subcase 1.1. Assume $u \in S_{0}$. Fix a hamiltonian path $\left(s_{i}\right)_{i=1}^{n}$ in $\operatorname{Cay}\left(\bar{G} /\left\langle\overline{S_{0}}\right\rangle ; S \backslash S_{0}\right)$ with $s_{1}=c$, and let $\pi_{i}=\prod_{j=1}^{i} s_{j}$. Any connected sum $C_{0} \#_{t_{1}}^{s_{1}}\left(-\pi_{1} C_{0}\right) \#_{t_{2}}^{s_{2}} \cdots \#_{t_{n}}^{s_{n}}\left( \pm \pi_{n} C_{0}\right)$ is a hamiltonian cycle $C$ in $\operatorname{Cay}(\bar{G} ; S)$.

Since $[t, c]$ and $[u, c]$ do not have the same projection to $\mathbb{Z}_{2}$, the voltages of $C_{0} \#_{t}^{c}$ $-\pi_{1} C_{0}$ and $C_{0} \#_{u}^{c}-\pi_{1} C_{0}$ do not have the same projection to $\mathbb{Z}_{2}$. Therefore, by choosing $t_{1}$ to be the appropriate element of $\{t, u\}$, we may assume the projection of $\Pi C$ to $\mathbb{Z}_{2}$ is nontrivial (see Corollary 3.14). Note also that if $\left|\bar{G}:\left\langle\overline{S_{0}}\right\rangle\right|=2$, then we must have $t_{1}=t$. 
We may assume that $t_{n}=s$, and that the connected sum $(-1)^{n-1} \pi_{n-1} C_{0} \#_{s}^{s_{n}}$ $(-1)^{n} \pi_{n} C_{0}$ is relative to the oriented edge $\left[\overline{\pi_{n} g}\right](s)$ of $\pi_{n} C_{0}$ that is also in $\pi_{n} C_{1}$. Therefore, another hamiltonian cycle $C^{\prime}$ can be constructed by replacing $\pi_{n} C_{0}$ with $\pi_{n} C_{1}$ in the connected sum. Then Lemma 3.12 (together with Lemma 2.6(2)) implies that $(\Pi C)^{-1}\left(\Pi C^{\prime}\right)$ is conjugate to $\left(\Pi C_{0}\right)^{-1}\left(\Pi C_{1}\right)$, which is a generator of $\mathbb{Z}_{p}$. Therefore, either $\Pi C$ or $\Pi C^{\prime}$ generates $G^{\prime}$, as desired.

Subcase 1.2. Assume $u \notin S_{0}$. Let $S_{u}=\{u\} \cup S_{0}$, let $n=\left|\left\langle\overline{S_{u}}\right\rangle:\left\langle\overline{S_{0}}\right\rangle\right|-1$, let $\left(s_{i}\right)_{i=1}^{m}$ be a hamiltonian path in $\operatorname{Cay}\left(\bar{G} /\left\langle\overline{S_{u}}\right\rangle ; S \backslash S_{u}\right)$ with $s_{1}=c$, and let $\pi_{i}=\prod_{j=1}^{i} s_{j}$. (Since $S \backslash S_{0}$ is an irredundant generating set for $\bar{G} /\left\langle\overline{S_{0}}\right\rangle$, we have $m, n \geq 1$.) Any connected sum

$$
C_{u}=C_{0} \#_{t_{1}}^{u}-u C_{0} \#_{t_{2}}^{u} \cdots \#_{t_{n}}^{u} \pm u^{n} C_{0}
$$

is a hamiltonian cycle in Cay $\left(\left\langle\overline{S_{u}}\right\rangle ; S_{u}\right)$, so any connected sum

$$
C=C_{u} \#_{t_{1}^{\prime}}^{s_{1}}-\pi_{1} C_{u} \#_{t_{2}^{\prime}}^{s_{2}} \cdots \#_{t_{m}^{\prime}}^{s_{m}} \pm \pi_{m} C_{u}
$$

is a hamiltonian cycle in $\operatorname{Cay}(\bar{G} ; S)$.

Since $t \in S_{0}$, we know that $C_{0}$ contains more than one edge labeled $t^{ \pm 1}$, so $-u C_{0}$ has an edge labeled $t^{ \pm 1}$ that was not removed in the construction of the connected $\operatorname{sum} C_{0} \#_{t_{1}}^{u}$ $-\pi_{1} C_{0}$. Furthermore, the definition of the connected sum implies that $C_{0} \#_{t_{1}}^{u}-\pi_{1} C_{0}$ also contains an edge labeled $u$. Therefore, we may form connected sums

$$
C_{u} \#_{t^{ \pm 1}}^{c}-\pi_{1} C_{u} \text { and } C_{u} \#_{u}^{c}-\pi_{1} C_{u}
$$

without removing any of the edges of $C_{u}$. Since $[c, t]$ and $[c, u]$ do not have the same projection to $\mathbb{Z}_{2}$, the voltages of these two connected sums do not have the same projection to $\mathbb{Z}_{2}$ (see Corollary 3.14). Therefore, by choosing $t_{1}^{\prime}$ to be the appropriate element of $\left\{t^{ \pm 1}, u\right\}$, we may assume the projection of $\Pi C$ to $\mathbb{Z}_{2}$ is nontrivial.

We have

$$
\begin{aligned}
C=C_{u} \#_{t_{1}^{\prime}}^{s_{1}}-\pi_{1} C_{u} \#_{t_{2}^{\prime}}^{s_{2}} \cdots \#_{t_{m-1}^{\prime}}^{s_{m-1}} \pm \pi_{m-1} C_{u} \#_{t_{m}^{\prime}}^{s_{m}} \\
\left( \pm \pi_{m} C_{0} \#_{t_{1}}^{u} \pm \pi_{m} u C_{0} \#_{t_{2}}^{u} \cdots \#_{t_{n}}^{u} \pm \pi_{m} u^{n} C_{0}\right),
\end{aligned}
$$

so the proof can be completed almost exactly as in the final paragraph of Subcase 1.1 (by constructing another connected sum in which $\pi_{m} u^{n} C_{0}$ is replaced with $\pi_{m} u^{n} C_{1}$ ).

Case 2. Assume $[u, c]$ projects nontrivially to $\mathbb{Z}_{2}$, for every $u \in S \backslash\{c\}$. In particular, $[d, c]$ projects nontrivially to $\mathbb{Z}_{2}$, for every $d \in S \backslash\left(S_{0} \cup\{c\}\right)$. Since we may assume that Case 1 does not apply with $d$ in the place of $c$, we conclude that we may assume

$$
[u, d] \text { projects nontrivially to } \mathbb{Z}_{2} \text {, for all } d \in S \backslash S_{0} \text { and } u \in S \backslash\{d\} \text {. }
$$

Choose a hamiltonian path $\left(s_{i}\right)_{i=1}^{n}$ in $\operatorname{Cay}\left(\bar{G} /\left\langle\overline{S_{0}}\right\rangle ; S \backslash S_{0}\right)$. Any connected sum

$$
C=C_{0} \#_{t_{1}}^{s_{1}}-\pi_{1} C_{0} \#_{t_{2}}^{s_{2}} \cdots \#_{t_{n}}^{s_{n}} \pm \pi_{n} C_{0}
$$


is a hamiltonian cycle in $\operatorname{Cay}(\bar{G} ; S)$. Calculating modulo $\mathbb{Z}_{p}$, and letting $z$ be the nontrivial element of $\mathbb{Z}_{2}$, we have

$$
\begin{aligned}
\Pi C & \equiv \Pi C_{0} \cdot\left[s_{1}, t_{1}\right] \cdot \Pi\left(-\pi_{1} C_{0}\right) \cdots\left[s_{n}, t_{n}\right] \cdot \Pi\left( \pm \pi_{n} C_{0}\right) & & (\text { Corollary 3.14) } \\
& \equiv \Pi C_{0} \cdot z \cdot \Pi C_{0} \cdots z \cdot \Pi C_{0} & & (\text { Lemma 2.6(2) \& (3.15A) }) \\
& =\left(\Pi C_{0}\right)^{n+1} \cdot z^{n} & & \\
& \equiv z & & (n \text { is odd }) .
\end{aligned}
$$

The proof is now completed exactly as in the final paragraph of Subcase 1.1.

Corollary 3.16. Let $S_{0} \subseteq S, g \in G$, and $s \in S_{0}$. Assume $C_{0}$ and $C_{1}$ are oriented hamiltonian cycles in Cay $\left(\left\langle\overline{S_{0}}\right\rangle ; S_{0}\right)$, such that

- $\left(\Pi C_{0}\right)^{-1}\left(\Pi C_{1}\right)$ is a nontrivial element of $\mathbb{Z}_{p}$,

- $C_{0}$ and $C_{1}$ both contain the oriented edge $[\bar{g}](s)$,

- for every $x \in S_{0}, C_{0}$ contains at least two edges that are labelled either $x$ or $x^{-1}$, and

- $\mathbb{Z}_{2} \nsubseteq\left\langle S_{0}\right\rangle^{\prime}$.

Then there is a hamiltonian cycle $C$ in $\operatorname{Cay}(\bar{G} ; S)$, such that $\langle\Pi C\rangle=G^{\prime}$, so the Factor Group Lemma (2.8) yields a hamiltonian cycle in $\operatorname{Cay}(G ; S)$.

Proof. We may assume $[c, t] \in \mathbb{Z}_{p}$, for all $c \in S$ and $t \in S_{0}$. (Otherwise, we see from Corollary 3.8 that Lemma 3.15(2) applies.) Choose $c, d \in S$, such that $[c, d] \notin \mathbb{Z}_{p}$, let $S_{0}^{+}=S_{0} \cup\{d\}$, and let $r=\left|\left\langle S_{0}^{+}\right\rangle:\left\langle S_{0}\right\rangle\right|$. Any connected sum of the following form is a hamiltonian cycle in $\operatorname{Cay}\left(\left\langle S_{0}^{+}\right\rangle ; S_{0}^{+}\right)$:

$$
C=C_{0} \#_{s_{1}}^{d}-d C_{0} \#_{s_{2}}^{d} \ldots \#_{s_{r-1}}^{d} \pm d^{r-1} C_{0} .
$$

We may assume $s_{1}=s$, and that the connected sum $C_{0} \#_{s_{1}}^{d}-d C_{0}$ is formed by using the oriented edge $[\bar{g}](s)$ that is also in $C_{1}$. Therefore, a second hamiltonian cycle $C^{\prime}$ can be constructed by replacing the leftmost occurrence of $C_{0}$ with $C_{1}$. Then Corollary 3.8 implies that Lemma 3.15(2) applies (with $S_{0}^{+}, d, d, C$, and $C^{\prime}$ in the roles of $S_{0}, s, t, C_{0}$, and $C_{1}$, respectively).

\section{Case with $\bar{s}=\bar{t}$}

Case 4.1. Assume there exist $s, t \in S \cup S^{-1}$ with $\bar{s}=\bar{t}$ and $s \neq t$.

Proof. Write $t=s \gamma$ with $\gamma \in G^{\prime}$. We may assume $\langle\gamma\rangle=G^{\prime}$, for otherwise $|\gamma|$ is prime, so Corollary 2.9 applies with $N=\langle\gamma\rangle$. Note that the irredundance of $S$ implies $\langle S \backslash\{s\}\rangle$ and $\langle S \backslash\{t\}\rangle$ do not contain $\mathbb{Z}_{p}$. This implies that every element of $S \backslash\{s, t\}$ centralizes $\mathbb{Z}_{p}$. So $s$ and $t$ do not centralize $\mathbb{Z}_{p}$.

Let $m=|\bar{t}|$ and $n=|\bar{G}| / m$.

Subcase 4.1.1. Assume $|\bar{t}|>2$. Since $\bar{G}$ is abelian, it is easy to find a hamiltonian cycle $C=\left(t_{i}\right)_{i=1}^{m n}$ in $\operatorname{Cay}(\bar{G} ; S \backslash\{s\})$, such that $t_{1}=t_{2}=\cdots=t_{m-1}=t$. Since $\langle\Pi C\rangle \subseteq$ $\langle S \backslash\{s\}\rangle$, and $\mathbb{Z}_{p} \nsubseteq\langle S \backslash\{s\}\rangle$, we must have $\Pi C \in \mathbb{Z}_{2}$.

For each subset $I$ of $\{1, \ldots, m-1\}$, we define $C_{I}$ to be the hamiltonian cycle constructed from $C$ by changing $t_{i}$ to $s$ for all $i \in I$. The proof is completed by noting that $I$ may be chosen such that $\Pi C_{I}$ generates $G^{\prime}$, so the Factor Group Lemma (2.8) applies: 
- If $\Pi C=e$, let $I=\{1\}$.

- If $\Pi C$ is the nontrivial element of $\mathbb{Z}_{2}$, and $t$ does not invert $\mathbb{Z}_{p}$, then we may let $I=\{1,2\}$.

- If $\Pi C$ is the nontrivial element of $\mathbb{Z}_{2}$, and $t$ inverts $\mathbb{Z}_{p}$, then $|\bar{t}|$ is even, so we must have $|\bar{t}| \geq 4$. We may let $I=\{1,3\}$.

Subcase 4.1.2. Assume $|\bar{t}|=2$. (Since $t$ does not centralize $\mathbb{Z}_{p}$, this implies that $t$ inverts $\mathbb{Z}_{p}$.) Choose a hamiltonian cycle $\left(s_{i}\right)_{i=1}^{n}$ in $\operatorname{Cay}(\bar{G} /\langle\bar{t}\rangle ; S \backslash\{s, t\})$, and let

$$
C_{0}=\left(t, s_{i}\right)_{i=1}^{n}=\left(t_{j}\right)_{j=1}^{2 n} .
$$

Since $n=|\bar{G}| / 2$ is even (see Corollary 3.9) and $S \backslash\{s\}$ is an irredundant generating set of $\bar{G}$, it is easy to see that $C_{0}$ is a hamiltonian cycle in $\operatorname{Cay}(\bar{G} ; S \backslash\{s\})$. Note that $t_{i}=t$ whenever $i$ is odd, and that $\Pi C_{0} \in \mathbb{Z}_{2}$ (because $\mathbb{Z}_{p} \nsubseteq\langle S \backslash\{s\}\rangle$ ).

We may assume $n \geq 6$ (for otherwise $|G|=4 n p \leq 20 p$, so Theorem 2.3 applies). We construct a hamiltonian cycle $C_{1}$ from $C_{0}$ :

- If $\Pi C_{0}=e$, construct $C_{1}$ by changing $t_{1}$ to $s$.

- If $\Pi C_{0} \neq e$, construct $C_{1}$ by changing both $t_{1}$ and $t_{5}$ to $s$.

In each case, $\Pi C_{1}$ generates $G^{\prime}$. (To see this in the second case, note that $t_{2} t_{3} t_{4} t_{5}=s_{1} t s_{2} t$ centralizes $G^{\prime}$, because $t$ inverts $G^{\prime}$, and each $s_{i}$ centralizes $G^{\prime}$.) Therefore, the Factor Group Lemma (2.8) applies.

\section{Cases with $|\bar{a}|>2$ and $\bar{b} \notin\langle\bar{a}\rangle$}

Recall that the elements $a$ and $b$ of $S$ satisfy (3.3A) and (3.3B).

Case 5.1. Assume $|\bar{a}|>2, \bar{b} \notin\langle\bar{a}\rangle$, and there exists $c \in S$, such that $\mathbb{Z}_{2} \subseteq\langle[a, c]\rangle$. (It may be the case that $b=c$.)

Proof. Let $m=|\bar{a}|$ and $n=|\bar{G}:\langle\bar{a}\rangle|$. Since $\bar{b}, \bar{c} \notin\langle\bar{a}\rangle$ (and $\bar{G} /\langle\bar{a}\rangle$ is abelian), it is easy to find a hamiltonian cycle $\left(s_{i}\right)_{i=1}^{n}$ in $\operatorname{Cay}(\bar{G} /\langle\bar{a}\rangle ; S \backslash\{a\})$, such that $s_{n} \in\left\{c^{ \pm 1}\right\}$, and $s_{k}=b$ for some $k<n$. Since $\mathbb{Z}_{2} \subseteq\langle[a, c]\rangle$, we know $m$ and $n$ are both even (see Corollary 3.8). Since $n$ is even, we have the following (well-known) hamiltonian cycle $C_{0}$ in $\operatorname{Cay}(\bar{G} ; \bar{S})$ :

$$
C_{0}=\left(a,\left(a^{m-2}, s_{2 i-1}, a^{-(m-2)}, s_{2 i}\right)_{i=1}^{n / 2} \#, a^{-1},\left(s_{n-j}^{-1}\right)_{j=1}^{n-1}\right) .
$$
fore

Letting $\widehat{G}=G / \mathbb{Z}_{p}$, we have $\widehat{G}^{\prime}=\mathbb{Z}_{2}$, so $\widehat{a}^{m-2} \in Z(\widehat{G})$ (because $m$ is even). There-

$$
a^{m-2} s_{2 i-1} a^{-(m-2)} \equiv s_{2 i-1} \quad\left(\bmod \mathbb{Z}_{p}\right),
$$

so, calculating modulo $\mathbb{Z}_{p}$, we have

$\Pi C_{0} \equiv a \cdot\left(\prod_{i=1}^{n-1} s_{j}\right) \cdot a^{-1} \cdot\left(\prod_{i=1}^{n-1} s_{j}\right)^{-1} \equiv a \cdot s_{n}^{-1} \cdot a^{-1} \cdot s_{n}=\left[a^{-1}, s_{n}\right]=\left[a^{-1}, c^{ \pm 1}\right]$

which is nontrivial $\left(\bmod \mathbb{Z}_{p}\right)$. 
Recall that $s_{k}=b$. Let $g=\prod_{i=1}^{k-1} s_{i}$ and $\delta=(-1)^{k+1}$. Then $C_{0}$ contains both the oriented edge $[\overline{g b}]\left(b^{-1}\right)$ and the oriented path $\left[\overline{g a^{-2 \delta}}\right]\left(a^{\delta}, b, a^{-\delta}\right)$. So Lemma 2.12 (with $s=a^{\delta}, t=b, u=a^{\delta}$ and $h=g$ ) provides a hamiltonian cycle $C_{1}$, such that $\left(\Pi C_{0}\right)^{-1}\left(\Pi C_{1}\right)$ is conjugate to $\left[b^{-1}, a^{\delta}\right]\left[a^{\delta}, b^{-1}\right]^{a^{\delta}}$. Since $a$ centralizes $\mathbb{Z}_{2}$, but not $\mathbb{Z}_{p}$, this voltage is a generator of $\mathbb{Z}_{p}$.

Thus, either $\Pi C_{0}$ or $\Pi C_{1}$ generates $\mathbb{Z}_{2} \times \mathbb{Z}_{p}=G^{\prime}$, so the Factor Group Lemma (2.8) provides a hamiltonian cycle in $\operatorname{Cay}(G ; S)$.

Case 5.2. Assume $|\bar{a}|>2, \bar{b} \notin\langle\bar{a}\rangle$, and there does not exist $c \in S$, such that $\mathbb{Z}_{2} \subseteq\langle[a, c]\rangle$.

Proof. Choose $c, d \in S$ with $\mathbb{Z}_{2} \subseteq\langle[c, d]\rangle$. Let

$$
m=|\bar{a}|, n=|\langle\bar{S} \backslash\{\bar{d}\}\rangle| / m \text {, and } r=|\bar{G}| /(m n) .
$$

By assumption, we know $a \notin\{c, d\}$. Also, we may assume $d \neq b$ (after interchanging $c$ and $d$ if necessary). Then Corollary 3.5 tells us $r>1$. Furthermore, we see from Corollary 3.8 that the image of $c$ in $\bar{G} /\langle\bar{a}\rangle$ has even order, so $n$ is even.

Subcase 5.2.1. Assume $n>2$. It is not difficult to construct a hamiltonian cycle $\left(s_{i}\right)_{i=1}^{n}$ in Cay $(\langle\bar{S} \backslash\{\bar{d}\}\rangle /\langle\bar{a}\rangle ; \bar{S} \backslash\{\bar{a}, \bar{d}\})$, such that $s_{1}=b$ and $s_{k}=c^{ \pm 1}$ for some $k \notin\{1, n\}$. Then, since $n$ is even, we may define $C_{0}$ as in (5.1A), so $C_{0}$ is a hamiltonian cycle in $\operatorname{Cay}(\langle\bar{S} \backslash\{\bar{d}\}\rangle ; S \backslash\{d\})$.

Let $g=s_{1} s_{2} \cdots s_{k}$, and note that $C_{0}$ contains the oriented edges $[\bar{e}](a)$ and $[\bar{g}]\left(c^{\mp 1}\right)$. Since $\mathbb{Z}_{2} \subseteq\langle[c, d]\rangle$, but $\mathbb{Z}_{2} \nsubseteq\langle[a, d]\rangle$, we see from Lemma 3.12 that there is a connected sum

$$
C=C_{0} \#_{t_{1}}^{d}-d C_{0} \#_{t_{2}}^{d} \cdots \#_{t_{r-1}}^{d} \pm d^{r-1} C_{0},
$$

with $t_{1} \in\left\{a, c^{ \pm 1}\right\}$, such that $\mathbb{Z}_{2} \subseteq\langle\Pi C\rangle$. Note that $C$ is a hamiltonian cycle in $\operatorname{Cay}(\bar{G} ; S)$.

The cycle $C_{0}$ contains both $[\bar{b}]\left(b^{-1}\right)$ and $\left[\bar{a}^{-2}\right]\left(a, b, a^{-1}\right)$, and neither of these paths contains either the edge $[\bar{e}](a)$ or the edge $[\bar{g}]\left(c^{\mp 1}\right)$. Therefore, $C$ also contains both of these paths, so Lemma 2.12 (with $s=a, t=b, u=a$, and $h=e$ ) provides a hamiltonian cycle $C^{\prime}$ in $\operatorname{Cay}(\bar{G} ; S)$, such that $(\Pi C)^{-1}\left(\Pi C^{\prime}\right)$ is a conjugate of $\left[b^{-1}, a\right]\left[a, b^{-1}\right]^{a}$, which is a generator of $\mathbb{Z}_{p}$ (since $a$ centralizes $\mathbb{Z}_{2}$, but not $\mathbb{Z}_{p}$ ). Then either $\Pi C$ or $\Pi C^{\prime}$ generates $G^{\prime}$, so the Factor Group Lemma (2.8) applies.

Subcase 5.2.2. Assume $n=2$ and $r>2$. Since $n=2$ (and $\bar{b} \notin\langle\bar{a}\rangle$ ), we have $\langle\bar{a}, \bar{b}, \bar{d}\rangle=$ $\bar{G}$, so Corollary 3.5 implies $S=\{a, b, d\}$. (Therefore $b=c$, which means $\mathbb{Z}_{2} \subseteq\langle[b, d]\rangle$.) We have the following hamiltonian cycle in $\operatorname{Cay}(\langle\bar{a}, \bar{b}\rangle ; \bar{a}, \bar{b})$ :

$$
C_{0}=[\bar{e}]\left(a^{m-1}, b, a^{-(m-1)}, b^{-1}\right) .
$$

Using the oriented edge $[\bar{e}](a)$, we can form the connected sum $C_{0} \#_{a}^{d}-d C_{0}$. Then, since $d C_{0}$ contains both $[\overline{d b}]\left(b^{-1}\right)$ and $[\overline{d a b}]\left(a^{-1}\right)$, we can extend this to a connected sum

$$
C=C_{0} \#_{a}^{d}-d C_{0} \#_{t_{2}}^{d} \cdots \#_{t_{r-1}}^{d} \pm d^{r-1} C_{0},
$$

with $t_{2} \in\{a, b\}$, such that $\mathbb{Z}_{2} \subseteq\langle\Pi C\rangle$ (see Corollary 3.14). Since $C$ contains both $[\bar{b}]\left(b^{-1}\right)$ and $\left[\bar{a}^{-2}\right]\left(a, b, a^{-1}\right)$, we may argue as in the last paragraph of Subcase 5.2.1. Namely, Lemma 2.12 (with $s=a, t=b, u=a$, and $h=e$ ) provides a hamiltonian cycle $C^{\prime}$ in $\operatorname{Cay}(\bar{G} ; S)$, such that $(\Pi C)^{-1}\left(\Pi C^{\prime}\right)$ is a conjugate of $\left[b^{-1}, a\right]\left[a, b^{-1}\right]^{a}$, which is 
a generator of $\mathbb{Z}_{p}$. Then either $\Pi C$ or $\Pi C^{\prime}$ generates $G^{\prime}$, so the Factor Group Lemma (2.8) applies.

Subcase 5.2.3. Assume $n=r=2$. As in Subcase 5.2.2, we must have $S=\{a, b, d\}$ and $b=c\left(\right.$ so $\left.\mathbb{Z}_{2} \subseteq\langle[b, d]\rangle\right)$.

Subsubcase 5.2.3.1. Assume $m \neq 3$. Since $m=|\bar{a}|>2$ (by an assumption of this case), we have $m \geq 4$. We have the following hamiltonian cycle in $\operatorname{Cay}(\bar{G} ; S)$ :

$$
C_{0}=\left(d, b, a, b^{-1}, d^{-1}, a^{m-2}, d, a^{-(m-3)}, b, a^{m-3}, d^{-1}, a^{-(m-1)}, b^{-1}\right) .
$$

Since $a$ is central in $G / \mathbb{Z}_{p}$ (by an assumption of this case), we know that

$$
\Pi C_{0} \equiv d b b^{-1} d^{-1} d b d^{-1} b^{-1}=d b d^{-1} b^{-1}=\left[d^{-1}, b^{-1}\right] \equiv[d, b]=[d, c] \quad\left(\bmod \mathbb{Z}_{p}\right),
$$

so $\mathbb{Z}_{2} \subseteq\left\langle\Pi C_{0}\right\rangle$.

Note that $C_{0}$ contains both $[\overline{d a b}]\left(b^{-1}\right)$ and $\left[\bar{d} \bar{a}^{3}\right]\left(a^{-1}, b, a\right)$ (because $\left.m \geq 4\right)$, so applying Lemma 2.12 (with $s=a^{-1}, t=b, u=a^{-1}$ and $h=d a$ ) yields a hamiltonian cycle $C_{1}$ in $\operatorname{Cay}(G ; S)$, such that $\left(\Pi C_{0}\right)^{-1}\left(\Pi C_{1}\right)$ is a conjugate of $\left[b^{-1}, a^{-1}\right]\left[a^{-1}, b^{-1}\right]^{a^{-1}}$, which is a generator of $\mathbb{Z}_{p}$. Then either $\Pi C$ or $\Pi C^{\prime}$ generates $G^{\prime}$, so the Factor Group Lemma (2.8) applies.

Subsubcase 5.2.3.2. Assume $m=3$ and $d$ does not centralize $G^{\prime}$. Since the walk $\left(a^{-2}, b^{-1}, a^{2}\right)$ is a hamiltonian path in $\operatorname{Cay}(\langle\bar{a}, \bar{b}\rangle ; a, b)$, we have the following hamiltonian cycle in $\operatorname{Cay}(\bar{G} ; S)$ :

$$
C=\left(a^{-2}, b^{-1}, a^{2}, d^{-1}, a^{-2}, b, a^{2}, d\right)
$$

Note that

$$
\Pi C=\left(a^{-2} b^{-1} a^{2}\right) d^{-1}\left(a^{-2} b a^{2}\right) d=\left(b^{a^{2}}\right)^{-1} d^{-1}\left(b^{a^{2}}\right) d=\left[b^{a^{2}}, d\right] .
$$

Since $a^{2}$ does not invert $G^{\prime}$, we know that $b^{a^{2}} \not \equiv b^{a^{-2}}\left(\bmod \mathbb{Z}_{2}\right)$. Therefore, since $d$ does not centralize $G^{\prime}$, we may assume $\left[b^{a^{2}}, d\right] \not \equiv e\left(\bmod \mathbb{Z}_{2}\right)$ (by replacing $a$ with its inverse if necessary). Also, since $G^{\prime}$ is central modulo $\mathbb{Z}_{p}$, we have $\left[b^{a^{2}}, d\right] \equiv[b, d] \not \equiv e\left(\bmod \mathbb{Z}_{p}\right)$. Therefore, $\Pi C$ generates $G^{\prime}$, so the Factor Group Lemma (2.8) applies.

Subsubcase 5.2.3.3. Assume $m=3$ and $d$ centralizes $G^{\prime}$. Suppose $[b, d] \in \mathbb{Z}_{2}$. Let $\widehat{G}=G / \mathbb{Z}_{2}$ and $\widehat{H}=\langle\widehat{a}, \widehat{b}\rangle$. From Theorem 1.1 , we know there is a hamiltonian cycle in $\operatorname{Cay}(\widehat{H} ; a, b)$. Deleting an edge labeled $b^{ \pm 1}$ (and passing to the reverse and/or a translate if necessary) yields a hamiltonian path $L=\left(t^{i}\right)_{i=1}^{2 m p-1}$ in $\operatorname{Cay}(\widehat{H} ; a, b)$ from $\widehat{e}$ to $\widehat{b}$. Let

$$
C=\left(L^{-1}, d^{-1}, L, d\right) \text {. }
$$

Then

$$
\Pi C=\left[\prod_{i=1}^{2 m p-1} t_{i}, d\right] \in\left[b \mathbb{Z}_{2}, d\right]=\{[b, d]\},
$$

because $\mathbb{Z}_{2}$ is in the center of $G$. Since $[b, d] \in \mathbb{Z}_{2}$, this calculation implies that $C$ is a closed walk in $G / \mathbb{Z}_{2}=\widehat{G}$. So $C$ is a hamiltonian cycle in Cay $(\widehat{G} ; S)$. The calculation also implies that the Factor Group Lemma (2.8) applies, because $\langle[b, d]\rangle=\mathbb{Z}_{2}$.

We may now assume $[b, d] \notin \mathbb{Z}_{2}$. Therefore, since $d$ centralizes $G^{\prime}$, and $p^{2} \nmid 12=|\bar{G}|$, we see from Lemma 3.6 that $b$ does not centralize $G^{\prime}$. Also, we may assume $[a, d] \neq e$, for otherwise Lemma 2.13 applies with $s=d$ and $t=a$. However, we know $\mathbb{Z}_{2} \nsubseteq\langle[a, d]\rangle$ (by an assumption of this case). Therefore $\langle[a, d]\rangle=\mathbb{Z}_{p}$. So Subsubcase 5.2.3.2 applies after interchanging $b$ and $d$. 


\section{Cases with $\bar{b} \in\langle\bar{a}\rangle$}

Case 6.1. Assume $\bar{b} \in\langle\bar{a}\rangle$ and a does not invert $G^{\prime}$.

Proof. Let $m=|\bar{a}|$. We may assume (perhaps after replacing $b$ with its inverse) that we may write $b=a^{k} \gamma$ with $1 \leq k \leq m / 2$ and $\gamma \in G^{\prime}$. Assume $k \geq 2$, for otherwise Case 4.1 applies. This implies $m-1 \geq k+1$ (since $m=|\bar{a}| \geq 2 k \geq k+2$ ).

Subcase 6.1.1. Assume there exists $c \in S$, such that $\mathbb{Z}_{2} \subseteq\langle[a, c]\rangle$. Let $n=|\bar{G}:\langle\bar{a}\rangle|$. Note that Corollary 3.8 implies $m$ and $n$ are even, and $c \notin\langle\bar{a}\rangle$ (so $c \neq b)$.

Choose a hamiltonian cycle $\left(s_{i}\right)_{i=1}^{n}$ in $\operatorname{Cay}(\bar{G} /\langle\bar{a}\rangle ; S \backslash\{a, b\})$, such that $s_{n}=c$, and define $C_{0}$ as in (5.1A). Then $\left\langle\Pi C_{0}\right\rangle$ contains $\mathbb{Z}_{2}$ by the same calculation as in Case 5.1.

Since $m-1 \geq k+1$, we may construct a hamiltonian cycle $C_{1}$ in Cay $(\bar{G} ; S)$ by replacing the path $\left(a^{k+1}\right)$ at the start of $C_{0}$ with $\left(b, a^{-(k-1)}, b\right)$. Then

$$
\begin{array}{r}
\left(\Pi C_{1}\right)\left(\Pi C_{0}\right)^{-1}=b a^{-(k-1)} b a^{-(k+1)}=\left(a^{k} \gamma\right) a^{-(k-1)}\left(a^{k} \gamma\right) a^{-(k+1)}= \\
a^{k+1} \gamma^{a} \gamma a^{-(k+1)} .
\end{array}
$$

This is a generator of $\mathbb{Z}_{p}$, since $a$ inverts $\mathbb{Z}_{2}$, but not $\mathbb{Z}_{p}$. Hence, either $\Pi C_{0}$ or $\Pi C_{1}$ generates $G^{\prime}$, so the Factor Group Lemma (2.8) provides a hamiltonian cycle in Cay $(G ; S)$.

Subcase 6.1.2. Assume there does not exist $c \in S$, such that $\mathbb{Z}_{2} \subseteq\langle[a, c]\rangle$. Choose $c, d \in S$, such that $\mathbb{Z}_{2} \subseteq\langle[c, d]\rangle$. (It is possible that $b \in\{c, d\}$, but we know, by the assumption of this subcase, that $a \notin\{c, d\}$.) Let $n=|\langle\bar{a}, \bar{d}\rangle:\langle\bar{a}\rangle|$ and $r=|\bar{G}| /(m n)$. From Corollary 3.8 (and the assumption of this subcase), we know $n$ and $r$ are even.

We have the following hamiltonian cycle in $\operatorname{Cay}(\langle\bar{a}, \bar{d}\rangle ; a, d)$ :

$$
C_{0}=\left(\left(a,\left(a^{m-2}, d, a^{-(m-2)}, d\right)^{n / 2} \#, a^{-1}, d^{-(n-1)}\right) .\right.
$$

As in the final paragraph of Subcase 6.1.1, another hamiltonian cycle $C_{1}$ can be constructed by replacing the path $\left(a^{k+1}\right)$ at the start of $C_{0}$ with $\left(b, a^{-(k-1)}, b\right)$, and the calculation in Subcase 6.1.1 shows that $\left(\Pi C_{1}\right)\left(\Pi C_{0}\right)^{-1}$ generates $\mathbb{Z}_{p}$. Therefore, since $[c, d] \notin \mathbb{Z}_{p}$, but $[c, a] \in \mathbb{Z}_{p}$, we see that Lemma 3.15(1) applies (with $S_{0}=\{a, b, d\}, g=a^{-1}, s=t=d$, and $u=a$ ).

Case 6.2. Assume $\bar{b} \in\langle\bar{a}\rangle$ and a inverts $G^{\prime}$.

Proof. As in Case 6.1, we let $m=|\bar{a}|$ and write $b=a^{k} \gamma$ with $2 \leq k \leq m / 2$ and $\gamma \in G^{\prime}$. We now consider the same five subcases as in [4, pp. 60-62].

Subcase 6.2.1. Assume $2<k<m / 2$ and $k$ is even. Let $C_{1}=\left(a^{m}\right)$. The proof in the last paragraph of $[4$, p. 60] provides a hamiltonian cycle

$$
C_{0}=\left(b, a^{-(k-4)}, b, a^{m-2 k-2}, b, a^{-1}, b, a^{2}, b^{-2}, a^{k-3}\right)
$$

in Cay $(\langle\bar{a}\rangle ; a, b)$, such that $\left(\Pi C_{0}\right)^{-1}\left(\Pi C_{1}\right)$ is a generator of $\mathbb{Z}_{p}$. Therefore, Corollary 3.16 applies (with $S_{0}=\{a, b\}$ ), because $C_{0}$ and $C_{1}$ both contain the oriented edge $\left[\bar{a}^{-1}\right](a)$.

Subcase 6.2.2. Assume $2<k<m / 2$ and $k$ is odd. Let

$$
C_{0}=\left(\left(b, a, b^{-1}, a\right)^{(k-1) / 2}, b, a^{m-2 k+1}\right)
$$


and

$$
C_{1}=\left(\left(b, a^{-1}, b^{-1}, a^{-1}\right)^{(k-1) / 2}, b^{2}, a^{m-2 k-1}, b\right) .
$$

Calculations in [4, p. 61] show that $\left(\Pi C_{0}\right)^{-1}\left(\Pi C_{1}\right)$ is a generator of $\mathbb{Z}_{p}$. Therefore, Corollary 3.16 applies (with $S_{0}=\{a, b\}$ ), because $C_{0}$ and $C_{1}$ both contain the oriented edge $[\bar{e}](b)$.

Subcase 6.2.3. Assume $k=m / 2$ and $k$ is even. We follow the argument of [11, Subcase iii, p. 97]. Since $k$ is even, we know $a^{k}$ centralizes $G^{\prime}$, so

$$
b^{2}=\left(a^{k} \gamma\right)^{2}=a^{2 k} \gamma^{2}=a^{m} \gamma^{2} \in \mathbb{Z}_{2} \cdot \gamma^{2} \not \supset e .
$$

Therefore Corollary 2.9 applies (with $s=b$ and $t=b^{-1}$ ).

Subcase 6.2.4. Assume $k=m / 2$ and $k$ is odd. Choose $c \in S$ so that $\mathbb{Z}_{2} \subseteq\langle[a, c]\rangle$, if such $c$ exists. Otherwise, choose $c$ so that there exists $d \in S$, such that $\mathbb{Z}_{2} \subseteq\langle[c, d]\rangle$. In either case, Corollary 3.8 implies $c \in S \backslash\{a, b\}$, and $|\langle\bar{a}, \bar{c}\rangle:\langle\bar{a}\rangle|$ is even.

We may assume $b^{2}=e$, for otherwise Corollary 2.9 applies (with $s=b$ and $t=b^{-1}$ ). Therefore, noting that $a^{k}$ inverts $G^{\prime}$ (since $k$ is odd), we have

$$
e=b^{2}=\left(a^{k} \gamma\right)\left(a^{k} \gamma\right)=a^{2 k} \cdot \gamma^{-1} \gamma=a^{m}
$$

Subsubcase 6.2.4.1. Assume $|\bar{G}:\langle\bar{a}\rangle|>2$. It suffices to find a hamiltonian cycle $C_{*}$ in Cay $(\bar{G} ; S)$, such that $\Pi C_{*}$ projects nontrivially to $\mathbb{Z}_{2}$, and $C_{*}$ contains the paths $\left[\overline{a^{k-3}}\right]\left(a, b, a^{-1}\right)$ and $\left[\overline{a^{k-1} b}\right]\left(b^{-1}\right)$. For then Lemma 2.12 (with $s=a, t=b, u=a$, and $\left.h=a^{k-1}\right)$ provides a hamiltonian cycle $C_{*}^{\prime}$, such that $\left\langle\left(\Pi C_{*}\right)^{-1}\left(\Pi C_{*}^{\prime}\right)\right\rangle=\mathbb{Z}_{p}$. Therefore, either $\Pi C_{*}$ or $\Pi C_{*}^{\prime}$ generates $G^{\prime}$, so the Factor Group Lemma (2.8) applies.

Note that

$$
C=\left(a^{k-2}, b, a^{-(k-2)}, c, a^{k-1}, c^{-1}, b^{-1}, c, a^{-(k-1)}, c^{-1}\right)
$$

is a cycle through the vertices of $\operatorname{Cay}(\bar{G} ;\{a, b, c\})$ in $\langle\bar{a}\rangle \cup c\langle\bar{a}\rangle$. A connected sum of translates of $C$ yields a hamiltonian cycle $C_{0}$ in Cay $(\bar{G} ; S)$.

If $\mathbb{Z}_{2} \nsubseteq\langle[a, c]\rangle$, then the connected sum defining $C_{0}$ can be chosen so that $\mathbb{Z}_{2} \subseteq\left\langle\Pi C_{0}\right\rangle$ (see the proof of Lemma 3.15). So we may let $C_{*}=C_{0}$.

We may now assume $\mathbb{Z}_{2} \subseteq\langle[a, c]\rangle$. Construct a hamiltonian cycle $C_{1}$ in $\operatorname{Cay}(\bar{G} ; S)$ by replacing the rightmost translate of $C$ in the connected sum with

$$
C^{\prime}=\left(a^{k-1}, b, a^{-(k-1)}, c, a^{k-1}, b^{-1}, a^{-(k-1)}, c^{-1}\right) .
$$

A straightforward calculation shows that $(\Pi C)^{-1}\left(\Pi C^{\prime}\right) \notin \mathbb{Z}_{p}$, so we have $\mathbb{Z}_{2} \subseteq\left\langle\Pi C_{i}\right\rangle$ for some $i \in\{0,1\}$. Let $C_{*}=C_{i}$.

Assumptions 6.2.4.2. We may now assume $|\bar{G}:\langle\bar{a}\rangle|=2$, so the irredundance of $S$ implies $S=\{a, b, c\}$. Since $\bar{b} \in\langle\bar{a}\rangle$, the irredundance of $S$ also implies $\langle[a, c]\rangle=\mathbb{Z}_{2}$. Furthermore, we may also assume that $c$ either centralizes $G^{\prime}$ or inverts $G^{\prime}$. (Otherwise, a preceding case applies after interchanging $a$ with $c$.)

Subsubcase 6.2.4.3. Assume c inverts $G^{\prime}$. Let

$$
L=\left\{\begin{array}{ll}
(a, b)^{k} \# & \text { if } p \mid k \\
(b, a)^{k} \# & \text { if } p \nmid k
\end{array} \quad \text { and } \quad C=\left(L^{-1}, c^{-1}, L, c\right) .\right.
$$


Then $L$ is a hamiltonian path in $\operatorname{Cay}(\langle\bar{a}\rangle ; a, b)$, so $C$ is a hamiltonian cycle in $\operatorname{Cay}(\bar{G} ; S)$. Since $(a b)^{k}=\gamma^{k}$, we have

$$
\Pi L= \begin{cases}\gamma^{k} b^{-1}=\gamma^{k-1} a^{-k} & \text { if } p \mid k, \\ \gamma^{-k} a^{-1} & \text { if } p \nmid k .\end{cases}
$$

Thus, in either case, we have $\Pi L=\gamma^{y} a^{z}$, where $p \nmid y$ and $z$ is odd, so

$$
\begin{aligned}
\Pi C & =(\Pi L)^{-1} c^{-1}(\Pi L) c=[\Pi L, c]=\left[\gamma^{y} a^{z}, c\right] \\
& =\left[\gamma^{y}, c\right]^{a^{z}} \cdot\left[a^{z}, c\right]=\left(\gamma^{-2 y}\right)^{a^{z}} \cdot[a, c]^{z}=\gamma^{2 y} \cdot[a, c] .
\end{aligned}
$$

This generates $G^{\prime}$, so the Factor Group Lemma (2.8) applies.

Subsubcase 6.2.4.4. Assume c centralizes $G^{\prime}$ and $k \geq 5$. Let

$$
C_{0}=\left(L, c, L^{-1}, c^{-1}\right),
$$

where $L=(b, a)^{k} \#$. Since $C_{0}$ contains both $[\bar{e}](b, a, b)$ and $[\bar{a} \bar{b} \bar{c}]\left(a^{-1}\right)$, and also contains both $\left[\bar{a}^{2}\right](b, a, b)$ and $\left[\bar{a}^{3} \bar{b} \bar{c}\right]\left(a^{-1}\right)$ we can apply Lemma 2.12 twice (first with $s=b, t=a$, $u=c$, and $h=b c$, and then with $s=b, t=a, u=c$, and $h=a^{2} b c$ ), to obtain a hamiltonian cycle $C_{2}$, such that

$$
\left(\Pi C_{0}\right)^{-1}\left(\Pi C_{2}\right)=\left[a^{-1}, b\right]^{2},
$$

which generates $\mathbb{Z}_{p}$. Then, since

$$
\Pi C_{0}=\left((b a)^{k} a^{-1}\right) c\left((b a)^{k} a^{-1}\right)^{-1} c^{-1}=[a, c]
$$

is a generator of $\mathbb{Z}_{2}$, we conclude that $\Pi C_{2}$ generates $G^{\prime}$, so the Factor Group Lemma (2.8) applies.

Subsubcase 6.2.4.5. Assume centralizes $G^{\prime}$ and $k=3$. Assume, for the moment, that $\gamma \notin \mathbb{Z}_{p}$. Let

$$
C=\left(c, b, c^{-1}, a, b^{-1}, c, b, a, b^{-1}, c^{-1}, b, a\right) .
$$

Then $C$ is a hamiltonian cycle in $\operatorname{Cay}(\bar{G} ; S)$, and a straightforward calculation shows that $\Pi C=b a^{3}=\gamma^{-1}$ generates $G^{\prime}$, so the Factor Group Lemma (2.8) applies.

Now, suppose that $p \geq 5$, and, because of the preceding paragraph, that $\gamma \in \mathbb{Z}_{p}$. Let

$$
C=\left(b, a, b^{-1}, a, b, c, a^{-5}, c^{-1}\right) .
$$

Then $C$ is a hamiltonian cycle in $\operatorname{Cay}(\bar{G} ; S)$ and

$$
\Pi C=b a b^{-1} a b c a c^{-1}=b a b^{-1} a b a\left[a, c^{-1}\right]=\gamma^{-3}[a, c] .
$$

Therefore $\langle\Pi C\rangle=G^{\prime}$ (since $p \neq 3$ and $\gamma$ projects trivially to $\mathbb{Z}_{2}$ ), so the Factor Group Lemma (2.8) applies.

We may now assume $p=3$ (so $|G|=72$ ), and that $\gamma \in \mathbb{Z}_{p}$. Let $\widehat{G}=G / \mathbb{Z}_{p}$. We have the following hamiltonian cycle in $\operatorname{Cay}(\widehat{G} ; S)$ :

$$
C=\left(a^{2}, c, a^{5}, c^{-1}, a^{-2}, b, a^{2}, c, a^{-5}, c^{-1}, a^{-2}, b\right) .
$$


Calculating modulo $\mathbb{Z}_{2}$ (so $c$ is in the center), we have

$$
\text { П } C=a^{2} c a^{5} c^{-1} a^{-2} b a^{2} c a^{-5} c^{-1} a^{-2} b \equiv a^{2} a^{5} a^{-2} b a^{2} a^{-5} a^{-2} b=a^{-1} b a b=[a, b]=\gamma^{2} .
$$

This is nontrivial $\left(\bmod \mathbb{Z}_{2}\right)$, so $\Pi C$ must be nontrivial. Therefore $\Pi C$ generates $\mathbb{Z}_{p}$, so the Factor Group Lemma (2.8) applies.

Subcase 6.2.5. Assume $k=2<m / 2$.

Subsubcase 6.2.5.1. Assume $|\bar{G}:\langle\bar{a}\rangle|>2$. Note that

$$
C=\left(b, a, b^{-1}, c, b, a^{-1}, b, c^{-1},\left(a, c, a, c^{-1}\right)^{(m-4) / 2}\right)
$$

is a cycle through the vertices of $\operatorname{Cay}(\bar{G} ;\{a, b, c\})$ in $\langle\bar{a}\rangle \cup c\langle\bar{a}\rangle$. A connected sum of translates of $C$ yields a hamiltonian cycle $C_{0}$ in $\operatorname{Cay}(\bar{G} ; S)$. Since $k$ is even, we know that $\mathbb{Z}_{2} \nsubseteq\langle[b, c]\rangle$, so it is easy to choose the connected sum in such a way that $\mathbb{Z}_{2} \subseteq\left\langle\Pi C_{0}\right\rangle$ (see the proof of Lemma 3.15).

The cycle $C$ contains the paths $[\bar{e}]\left(b, a, b^{-1}\right)$ and $\left[\bar{b}^{2}\right](a)$. By taking just a bit of care in the creation of $C_{0}$ (namely, not using any of these edges for the first connected sum), we may assume that $C_{0}$ also contains these paths. Then Lemma 2.12 (with $s=b, t=a$, $u=b$, and $\left.h=b^{2}\right)$ provides a hamiltonian cycle $C_{1}$, such that $\left(\Pi C_{0}\right)^{-1}\left(\Pi C_{1}\right)=[a, b]^{2}$ (because $b$ centralizes $G^{\prime}$ ). This is a generator of $\mathbb{Z}_{p}$, so either $\Pi C_{0}$ or $\Pi C_{1}$ generates $G^{\prime}$. Therefore, the Factor Group Lemma (2.8) applies.

Subsubcase 6.2.5.2. Assume $|\bar{G}:\langle\bar{a}\rangle|=2$. The irredundance of $S$ implies that $S=$ $\{a, b, c\}$ (see Corollary 3.5). We have the following hamiltonian cycle in $\operatorname{Cay}(\bar{G} ; S)$ :

$$
C=\left(b^{2}, a^{m-5}, c, a^{-(m-4)}, c^{-1}, b^{-1}, c, a, b^{-1}, c^{-1}\right) .
$$

Since $\bar{b} \in\langle\bar{a}\rangle$, the irredundance of $S$ implies $\langle[a, c]\rangle=\mathbb{Z}_{2}$. So $m$ is even (see Corollary 3.8). However, $\mathbb{Z}_{2} \nsubseteq\langle[b, c]\rangle$, because $k=2$ is even. So

$$
\begin{aligned}
\Pi C & =b^{2}\left(a^{m-5} c a^{-(m-4)} c^{-1}\right)\left(b^{-1} c a b^{-1} c^{-1}\right) \\
& \equiv b^{2}\left(a^{-1}\right)\left(b^{-2} a[a, c]\right) \equiv[a, c] \quad\left(\bmod \mathbb{Z}_{p}\right),
\end{aligned}
$$

which generates $\mathbb{Z}_{2}$. We may also assume that $c$ either centralizes $G^{\prime}$ or inverts $G^{\prime}$ (for otherwise a preceding case applies after interchanging $a$ with $c$ ). Therefore

$$
\begin{aligned}
\Pi C & =b^{2}\left(a^{m-5} c a^{-(m-4)} c^{-1}\right)\left(b^{-1} c a b^{-1} c^{-1}\right) \equiv a^{4} \gamma^{2}\left(a^{-1}\right)\left(\gamma^{-1} a^{-2} c a \gamma^{-1} a^{-2} c^{-1}\right) \\
& =\gamma^{3} \cdot\left(\gamma^{-1}\right)^{c}=\gamma^{3} \cdot \gamma^{ \pm 1} \in\left\{\gamma^{2}, \gamma^{4}\right\} \quad\left(\bmod \mathbb{Z}_{2}\right),
\end{aligned}
$$

which generates $\mathbb{Z}_{p}$. We now know that $\Pi C$ projects nontrivially to both $\mathbb{Z}_{2}$ and $\mathbb{Z}_{p}$, so it generates $G^{\prime}$. Therefore, the Factor Group Lemma (2.8) applies.

\section{Cases with $|\bar{a}|=2$ and $\# S=2$}

Assumption 7.1. In this section, we assume

- $|\bar{a}|=2$, for all $a \in S$, such that $a$ does not centralize $G^{\prime}$, and

- $\# S=2$. 
We may assume $|a|=2$, for otherwise Case 4.1 applies with $s=a$ and $t=a^{-1}$.

We may also assume that $b$ centralizes $G^{\prime}$, for otherwise we must have $|\bar{b}|=2$, so $|G|=8 p$, so Theorem 2.3 applies. Since $a$ does not centralize $G^{\prime}$, this implies $\bar{a} \notin\langle\bar{b}\rangle$. Let

$$
n=|\bar{G}:\langle\bar{a}\rangle|=|\bar{G}| / 2=|\bar{b}| .
$$

Case 7.2. Assume $n \not \equiv 1(\bmod p)$.

Proof. Let $C=\left(a^{-1}, b^{-(n-1)}, a, b^{n-1}\right)$, so $C$ is a hamiltonian cycle in $\operatorname{Cay}(\bar{G} ; S)$ with $\Pi C=\left[a, b^{n-1}\right]=[a, b]^{n-1}$, since $b$ centralizes $G^{\prime}$. Note that $n$ is even (see Corollary 3.8), and, by assumption, $n \not \equiv 1(\bmod p)$. Therefore, $n-1$ is relatively prime to $2 p$, so $\Pi C$ generates $G^{\prime}$, so the Factor Group Lemma (2.8) applies.

Case 7.3. Assume $n \equiv 1(\bmod p)$.

Proof. We claim that $\mathbb{Z}_{p} \subseteq\langle b\rangle$. Suppose not. Then $\left|\left\langle b, \mathbb{Z}_{2}\right\rangle\right|=2 n$. Since $\operatorname{gcd}(2 n, p)=1$, the abelian group $\left\langle b, G^{\prime}\right\rangle$ has a unique subgroup of order $2 n$, so we conclude that $\left\langle b, \mathbb{Z}_{2}\right\rangle$ is normal in $G$. This implies that

$$
\langle a\rangle\left\langle b, \mathbb{Z}_{2}\right\rangle=\left\langle a, b, \mathbb{Z}_{2}\right\rangle \supseteq\langle a, b\rangle=G,
$$

so

$$
|G| \leq|a| \cdot\left|\left\langle b, \mathbb{Z}_{2}\right\rangle\right|=2 \cdot 2 n=4 n .
$$

This contradicts the fact that $|G|=4 n p$.

Subcase 7.3.1. Assume $\mathbb{Z}_{2} \subseteq\langle b\rangle$. Combining this assumption with the above claim, we see that $G^{\prime} \subseteq\langle b\rangle$. This implies $\langle b\rangle \triangleleft G$, so $G=\langle a\rangle \ltimes\langle b\rangle$. Since $|a|=2$, this implies that $\operatorname{Cay}(G ; a, b)$ is a generalized Petersen graph. Then the main result of [1] tells us that $\operatorname{Cay}(G ; a, b)$ has a hamiltonian cycle.

Subcase 7.3.2. Assume $\mathbb{Z}_{2} \nsubseteq\langle b\rangle$. Since $\left\langle b, G^{\prime}\right\rangle$ is abelian, $\operatorname{gcd}(n, p)=1$, and $\mathbb{Z}_{2} \nsubseteq\langle b\rangle$, we may write

$$
\left\langle b, G^{\prime}\right\rangle=\mathbb{Z}_{2} \times \mathbb{Z}_{p} \times \mathbb{Z}_{n} .
$$

Then $G=\langle a\rangle \ltimes\left(\mathbb{Z}_{2} \times \mathbb{Z}_{p} \times \mathbb{Z}_{n}\right)$, and we may assume $b=(0,1,1)$ and $[a, b]=(1,2,0)$. For $\underline{G}=G /\left\langle b^{2}\right\rangle=G /\left(\mathbb{Z}_{p} \times 2 \mathbb{Z}_{n}\right)$, it is straightforward to check that $\left((a, b)^{4} \#, b^{-1}\right)$ is a hamiltonian cycle in $\operatorname{Cay}(\underline{G} ; a, b)$ whose voltage is $(0,-2,2)$. (This hamiltonian cycle is taken from the final paragraph of Case 1 of the proof of [3, Prop. 6.1].) This voltage generates $\mathbb{Z}_{p} \times 2 \mathbb{Z}_{n}($ since $\operatorname{gcd}(p, n)=1)$, so the Factor Group Lemma (2.8) applies.

\section{Cases with $|\bar{a}|=2$ and $\# S=3$}

Assumption 8.1. In this section, we assume

$$
S=\{a, b, c\}
$$

and

$$
|\bar{s}|=2 \text {, for all } s \in S \text {, such that } s \text { does not centralize } G^{\prime} .
$$

We also assume Case 4.1 does not apply. (So $|s|=2$.) In particular, we have $|a|=2$.

Note that $\bar{a} \notin\langle\bar{b}\rangle$. (If $\bar{a} \in\langle\bar{b}\rangle$, then $b$, like $a$, does not centralize $G^{\prime}$, so our assumption implies $|\bar{b}|=2$. Then $\bar{a}=\bar{b}$, contradicting the fact that Case 4.1 does not apply.) 
Notation 8.2. Let

$$
n=|\bar{b}|=|\langle\bar{a}, \bar{b}\rangle:\langle\bar{a}\rangle| \geq 2 \quad \text { and } \quad \ell=|\bar{G}:\langle\bar{a}, \bar{b}\rangle|=|\bar{G}| /(2 n) \geq 2 .
$$

The last inequality is because the irredundance of $S$ implies $\bar{c} \notin\langle\bar{a}, \bar{b}\rangle$ (see Corollary 3.5).

Case 8.3. Assume $|\bar{b}|=3$.

Proof. Since $|\bar{b}| \neq 2$, Assumption 8.1 implies that $b$ centralizes $G^{\prime}$. Also, since $|\bar{b}|$ is odd, Corollary 3.8 implies that $[a, b]$ and $[b, c]$ project trivially to $\mathbb{Z}_{2}$, so $[a, c]$ must project nontrivially (and $\ell$ must be even). We have the following hamiltonian path in $\operatorname{Cay}(\bar{G} /\langle\bar{a}\rangle ; S)$ :

$$
L=\left(c^{\ell-1}, b, c^{-(\ell-1)}, b, c^{\ell-1}\right) .
$$

Then $C=\left(L, a, L^{-1}, a\right)$ is a hamiltonian cycle in Cay $(\bar{G} ; S)$. Since $\ell-1$ is odd, it is easy to see that $\mathbb{Z}_{2} \subseteq\langle\Pi C\rangle$.

Since $C$ contains both $\left[\bar{c}^{\ell-2}\right]\left(c, b, c^{-1}\right)$ and $\left[\bar{c}^{\ell-1} \bar{a} \bar{b}\right]\left(b^{-1}\right)$, Lemma 2.12 (with $s=c$, $t=b, u=a$, and $\left.h=c^{\ell-1} a\right)$ provides a hamiltonian cycle $C^{\prime}$, such that $(\Pi C)^{-1}\left(\Pi C^{\prime}\right)$ is conjugate to $\left[t^{-1}, u\right]\left[s, t^{-1}\right]^{u}=\left[b^{-1}, a\right]\left[c, b^{-1}\right]^{a}=[a, b][c, b]$. This is an element of $\mathbb{Z}_{p}$. If it generates $\mathbb{Z}_{p}$, then either $\Pi C$ or $\Pi C^{\prime}$ generates $G^{\prime}$, so the Factor Group Lemma (2.8) applies.

Thus, we may assume $[a, b][c, b]$ is trivial. Since $\mathbb{Z}_{p} \subseteq\langle[a, b]\rangle$ (see (3.3B)), this implies that $[c, b]$ is nontrivial. So we may assume that $c$ does not centralize $\mathbb{Z}_{p}$ (for otherwise replacing $c$ with $c^{-1}$ would replace $[c, b]$ with $[c, b]^{-1}$, which would not cancel $[a, b]$ ).

Now, Assumption 8.1 implies $|\bar{c}|=2$, so we have the hamiltonian cycle

$$
C_{0}=\left(b^{2}, a, b^{2}, c, a, b, a, b, a, c\right),
$$

in $\operatorname{Cay}(\bar{G} ; S)$. This contains both the path $[b a c](a, b, a)$ and the edge $[b](b)$, so applying Lemma 2.12 (with $s=a, t=b, u=c$, and $h=b$ ) provides a hamiltonian cycle $C_{1}$, such that $\left(\Pi C_{0}\right)^{-1}\left(\Pi C_{1}\right)$ is conjugate to $\left[u, t^{-1}\right]\left[s, t^{-1}\right]^{u}=\left[c, b^{-1}\right]\left[a, b^{-1}\right]^{c}$. This is not equal to $[a, b][c, b]$ (which is trivial), because $\left[a, b^{-1}\right]^{c}=[a, b]$, but $\left[c, b^{-1}\right]=[c, b]^{-1} \neq$ $[c, b]$. So $\left(\Pi C_{0}\right)^{-1}\left(\Pi C_{1}\right)$ is nontrivial, and therefore generates $\mathbb{Z}_{p}$. Since a straightforward calculation shows that $\mathbb{Z}_{2}$ is contained in $\left\langle\Pi C_{0}\right\rangle$, this implies that either $\Pi C_{0}$ or $\Pi C_{1}$ generates $G^{\prime}$, so the Factor Group Lemma (2.8) applies.

Case 8.4. Assume $\ell=2$.

Proof. We may assume $|\bar{b}| \geq 4$, for otherwise either $|\bar{b}|=2$, so Theorem 2.3 applies (because $|G|=16 p$ ), or $|\bar{b}|=3$, so Case 8.3 applies. Let

$$
L=\left(a, b, a, b^{n-2}, a, b^{-(n-3)}\right) \quad \text { and } \quad C=\left(L, c, L^{-1}, c^{-1}\right),
$$

so $L$ is a hamiltonian path in $\operatorname{Cay}(\langle\bar{a}, \bar{b}\rangle ; a, b)$ and $C$ is a hamiltonian cycle in $\operatorname{Cay}(\bar{G} ; S)$.

Subcase 8.4.1. Assume $[a, c]$ and $[a, b][b, c]$ are not both in $\mathbb{Z}_{p}$. A straightforward calculation (using Lemma 3.6) shows that $\Pi C \equiv[a, c]\left(\bmod \mathbb{Z}_{p}\right)$. If this is in $\mathbb{Z}_{p}$, then, by assumption, $[a, b][b, c] \notin \mathbb{Z}_{p}$, so applying Lemma 2.12 to the paths $[\bar{e}](a, b, a)$ and $[\overline{a b c}]\left(b^{-1}\right)$ in $C$ (so $s=a, t=b, u=c$, and $h=a c$ ) yields a hamiltonian cycle $C^{\prime}$, such that $\Pi C^{\prime}$ projects nontrivially to $\mathbb{Z}_{2}$. Therefore, we have a hamiltonian cycle (either $C$ or $C^{\prime}$ ) whose voltage is not in $\mathbb{Z}_{p}$. 
Now, since $|\bar{b}| \geq 4$, we see that $C$ (and also $C^{\prime}$ ) contains the path $\left[\overline{b^{-2} a c}\right]\left(b, a, b^{-1}\right.$ ) and $[\overline{a c}](a)$. Furthermore, we know that $[b, a][b, a]^{b}$ is a nontrivial element of $\mathbb{Z}_{p}$ (because $b$ does not invert $[a, b]$ ). Therefore, Lemma 2.12 (with $s=b, t=a, u=b$, and $h=a c$ ) yields a hamiltonian cycle $C_{1}$ (or $C_{1}^{\prime}$ ) whose voltage generates $G^{\prime}$, so the Factor Group Lemma (2.8) applies.

Subcase 8.4.2. Assume $[a, c]$ and $[a, b][b, c]$ are both in $\mathbb{Z}_{p}$. Since $[a, c],[a, b]$, and $[b, c]$ generate $G^{\prime}$, they cannot all be in $\mathbb{Z}_{p}$, so this assumption implies that neither $[a, b]$ nor $[b, c]$ is in $\mathbb{Z}_{p}$. Also, we may assume $\langle[a, c]\rangle=\mathbb{Z}_{p}$, for otherwise $[a, c]=e$, so we could apply Lemma 2.13 with $s=c$.

We have the following hamiltonian cycle in $\operatorname{Cay}(\bar{G} ; S)$ :

$$
C_{0}=\left(b^{n-1}, c, b^{-(n-2)}, a, b^{n-2}, c^{-1}, b^{-(n-1)}, c, a, c^{-1}\right) .
$$

Then

$$
\begin{aligned}
\Pi C_{0} & =b^{n-1} c\left(b^{-(n-2)} a b^{n-2}\right) c^{-1} b^{-(n-1)} c a c^{-1} \\
& =b^{n-1} c\left(a[a, b]^{n-2}\right) c^{-1} b^{-(n-1)} c a c^{-1} \\
& =\left([a, b]^{-(n-2)}\right)^{c} \cdot b^{n-1}\left(c a c^{-1}\right) b^{-(n-1)}\left(c a c^{-1}\right) \\
& =\left([a, b]^{-(n-2)}\right)^{c} \cdot\left[b, c a c^{-1}\right]^{-(n-1)} \\
& =\left([a, b]^{-(n-2)}\right)^{c} \cdot[b, a]^{-(n-1)} \\
& =\left([a, b]^{-(n-2)}\right)^{c} \cdot[a, b]^{n-1} .
\end{aligned}
$$

If $c$ centralizes $\mathbb{Z}_{p}$, then $\Pi C_{0}=[a, b]$ generates $G^{\prime}$, so the Factor Group Lemma (2.8) applies.

We may now assume $c$ does not centralize $\mathbb{Z}_{p}$. Then Assumption 8.1 tells us that $c$ inverts $\mathbb{Z}_{p}$, so $\Pi C_{0}=[a, b]^{2 n-3}$ (and $\left.|c|=2\right)$. Hence, we may assume $2 n \equiv 3(\bmod p)$, for otherwise $\Pi C_{0}$ generates $G^{\prime}$, so the Factor Group Lemma (2.8) applies. We now consider the following hamiltonian cycle in $\operatorname{Cay}(\bar{G} ; S)$ :

$$
C_{*}=\left(b^{n-3}, c, b^{-(n-4)}, a, b^{n-4}, c^{-1}, b^{-(n-3)}, c,\left(b^{-1}, c\right)^{2}, a,(c, b)^{2}, c^{-1}\right) .
$$

We have

$$
\Pi C_{*}=b^{n-3} c\left(b^{-(n-4)} a b^{n-4}\right) c^{-1} b^{-(n-3)} c\left(\left(b^{-1} c\right)^{2} a(c b)^{2}\right) c^{-1} .
$$

Since $c b$ inverts $G^{\prime}$, we know that $\left(b^{-1} c\right)^{2} a(c b)^{2}=a$, so $\Pi C_{*}$ is exactly the same as the voltage of $C_{0}$, but with $n$ replaced by $n-2$; that is,

$$
\Pi C_{*}=[a, b]^{2(n-2)-3}=[a, b]^{2 n-7} .
$$

Since $2 n \equiv 3(\bmod p)$, we have

$$
2 n-7 \equiv 3-7=-4 \not \equiv 0 \quad(\bmod p),
$$

so $\Pi C_{*}$ generates $G^{\prime}$, so the Factor Group Lemma (2.8) applies.

Case 8.5. Assume $|\bar{b}| \neq 3$ and $\ell \neq 2$. 
Proof. Since $\ell \neq 2$, we know $|\bar{c}|>2$, so $c$ must centralize $G^{\prime}$ (by Assumption 8.1). Also, Corollary 3.8 implies that $|\bar{b}|$ and $\ell$ cannot both be odd.

- If $|\bar{b}|$ is odd (so $\ell$ is even), let

$$
L=\left(c^{\ell-1}, b, c^{-1}, b, c, b,\left(b^{n-4}, c^{-1}, b^{-(n-4)}, c^{-1}\right)^{\ell / 2} \#, b^{-1}, c^{\ell-3}, b^{-1}, c^{-(\ell-3)}\right) .
$$

- If $|\bar{b}|$ is even, let

$$
L=\left(c^{\ell-1}, b^{n-1}, c^{-1},\left(c^{-(\ell-2)}, b^{-1}, c^{\ell-2}, b^{-1}\right)^{(n-2) / 2}, c^{-(\ell-2)}\right) .
$$

In either case, $L$ is a hamiltonian path in $\operatorname{Cay}(\bar{G} /\langle\bar{a}\rangle ;\{b, c\})$ from $\bar{e}$ to $\bar{b}$. Now, let

$$
C=\left(L, a, L^{-1}, a\right) \quad \text { and } \quad(g, \epsilon)= \begin{cases}\left(c^{\ell-1},-1\right) & \text { if }|\bar{b}|=2 \text { or }|\bar{b}| \text { is odd } \\ \left(a b^{2}, 1\right) & \text { if }|\bar{b}|>2 \text { and }|\bar{b}| \text { is even }\end{cases}
$$

so $C$ is a hamiltonian cycle in $\operatorname{Cay}(\bar{G} ; S)$ that contains the paths

$$
[\overline{b c}]\left(c^{-1}, a, c\right), \quad[\overline{c a}]\left(c^{-1}, a, c\right), \quad[\bar{g}](b), \quad \text { and } \quad\left[\overline{g b a c^{\epsilon}}\right]\left(c^{-\epsilon}, b^{-1}, c^{\epsilon}\right) .
$$

Note that $[\overline{b c}]\left(c^{-1}, a, c\right)$ contains $[\bar{b}](a)$ and that $[\overline{c a}]\left(c^{-1}, a, c\right)$ contains $[\bar{a}](a)$. Also note that all of these paths are vertex-disjoint (except for the vertices $\overline{a c}$ and $\{a b c\}$ when $|\bar{b}|=2$ and $\ell=3)$. We introduce some terminology:

- Applying Lemma 2.12 to the oriented paths $[\overline{c a}]\left(c^{-1}, a, c\right)$ and $[\bar{b}](a)$ (so $s=c^{-1}$, $t=a, u=b$, and $h=a b$ ) will be called the " $a$-transform." This multiplies the voltage by $\gamma_{a}$, where $\gamma_{a}=\left[a, b^{-1}\right][c, a]$.

- Applying Lemma 2.12 to the oriented paths $[\bar{g}](b)$ and $\left[\overline{g b a c^{\epsilon}}\right]\left(c^{-\epsilon}, b^{-1}, c^{\epsilon}\right)$ (so $s=$ $c^{-\epsilon}, t=b^{-1}, u=a$, and $h=g b$ ) will be called the " $b$-transform." This multiplies the voltage by a conjugate of $\gamma_{b}$, where $\gamma_{b}=[b, a]\left[b, c^{-\epsilon}\right]$.

Subcase 8.5.1. Assume precisely one of $\gamma_{a}$ and $\gamma_{b}$ is in $\mathbb{Z}_{p}$. Write $\{a, b\}=\{x, y\}$, such that $\gamma_{x} \in \mathbb{Z}_{p}$ and $\gamma_{y} \notin \mathbb{Z}_{p}$. We may assume $\left\langle\gamma_{x}\right\rangle=\mathbb{Z}_{p}$ (by replacing $c$ with its inverse, if necessary). Choose $C^{\prime}$ to be either $C$ or the $y$-transform of $C$, such that $\Pi C^{\prime}$ projects nontrivially to $\mathbb{Z}_{2}$. Then choose $C^{\prime \prime}$ to be either $C^{\prime}$ or the $x$-transform of $C^{\prime}$, such that $\Pi C^{\prime \prime}$ generates $G^{\prime}$, so the Factor Group Lemma (2.8) applies.

Subcase 8.5.2. Assume $\gamma_{a}$ and $\gamma_{b}$ are both in $\mathbb{Z}_{p}$. Since $[a, b],[a, c]$, and $[b, c]$ cannot all be in $\mathbb{Z}_{p}$, this assumption implies that none of them are in $\mathbb{Z}_{p}$. Therefore, since the path $L$ has odd length, we see that $\Pi C$ has nontrivial projection to $\mathbb{Z}_{2}$.

We may assume (by replacing $c$ with its inverse, if necessary), that $\gamma_{a}$ has nontrivial projection to $\mathbb{Z}_{p}$, so $\left\langle\gamma_{a}\right\rangle=\mathbb{Z}_{p}$. Therefore, by choosing $C^{\prime}$ to be either $C$ or the $a$-transform of $C$, such that $\Pi C^{\prime}$ generates $G^{\prime}$, we may apply the Factor Group Lemma (2.8).

Subcase 8.5.3. Assume neither $\gamma_{a}$ nor $\gamma_{b}$ is in $\mathbb{Z}_{p}$, and $b$ centralizes $G^{\prime}$. Note that the sum of the exponents of the occurrences of $b$ in $L$ is 1 , and the sum of the exponents of the occurrences of $c$ is 0 . Therefore, since $b$ and $c$ centralize $G^{\prime}$, Lemma 3.6 implies that $\Pi C=[a, b]$. Hence, we may assume $[a, b] \in \mathbb{Z}_{p}$ (for otherwise $\langle\Pi C\rangle=G^{\prime}$, so the Factor Group Lemma (2.8) applies). Then, by the assumption of this subcase, we conclude that 
$[a, c] \notin \mathbb{Z}_{p}$. So we may assume $\langle[a, c]\rangle=\mathbb{Z}_{2}$, for otherwise $b$ and $c$ could be interchanged, resulting in a situation in which $[a, b] \notin \mathbb{Z}_{p}$, and which has therefore already been covered. Also, since $[a, b] \in \mathbb{Z}_{p}$ and $[a, c] \notin \mathbb{Z}_{p}$, Corollary 3.8 tells us that $\ell$ is even (and recall that $\ell \neq 2$ ).

Since $[a, b]$ is a nontrivial element of $\mathbb{Z}_{p}$, and $b$ centralizes $G^{\prime}$, we see from Corollary 3.7 that $|b|$ is divisible by $p$. Therefore, $|b| \neq 2$, so we may assume $|\bar{b}|>2$ (for otherwise Case 4.1 applies with $s=b$ and $t=b^{-1}$ ). Since $|\bar{b}| \neq 3$ (by the assumption of this case), this implies $n=|\bar{b}| \geq 4$, so we may let

$$
L_{0}=\left(c^{\ell-1}, b, c^{-(\ell-1)}, b^{2},\left(b^{n-4}, c, b^{-(n-4)}, c\right)^{\ell / 2} \#, b^{-1}, c^{-(\ell-2)}\right),
$$

so $L_{0}$ is a hamiltonian path from $\bar{e}$ to $\overline{b^{2} c}$ in $\operatorname{Cay}(\bar{G} /\langle\bar{a}\rangle ;\{b, c\})$. Note that the sum of the exponents of the occurrences of $b$ in $L$ is 2 , and the sum of the exponents of the occurrences of $c$ is 1 . Therefore, since $b$ and $c$ centralize $G^{\prime}$, Lemma 3.6 implies $\Pi\left(L_{0}, a, L_{0}^{-1}, a\right)=$ $[a, b]^{2}[a, c]$. This generates $G^{\prime}$, so the Factor Group Lemma (2.8) applies.

Subcase 8.5.4. Assume neither $\gamma_{a}$ nor $\gamma_{b}$ is in $\mathbb{Z}_{p}$, and $b$ does not centralize $\mathbb{Z}_{p}$. From Assumption 8.1, we know $\bar{b}=2$ (so $b$ must invert $G^{\prime}$ ).

We may assume $[a, c] \in \mathbb{Z}_{2}$, for otherwise Case 8.4 could be applied by interchanging $b$ and $c$. Then we may assume $[a, c]$ is the nontrivial element of $\mathbb{Z}_{2}$, for otherwise the assumption that $\gamma_{a} \notin \mathbb{Z}_{p}$ implies $\langle[a, b]\rangle=G^{\prime}$, so $\langle a, b\rangle \triangleleft G$, and then Lemma 2.13 applies with $s=c$.

By applying the same argument, with $a$ and $b$ interchanged, we may assume $[b, c]$ is also the nontrivial element of $\mathbb{Z}_{2}$. This implies $[a, b] \in \mathbb{Z}_{p}$, since $\gamma_{b} \notin \mathbb{Z}_{p}$.

Note that, since $a$ and $b$ both have order 2 (and invert $G^{\prime}$ ), the image of $\langle a, b\rangle$ in $G / \mathbb{Z}_{2}$ is the dihedral group of order $2 p$. Also, the preceding two paragraphs imply that $c$ is in the center of $G / \mathbb{Z}_{2}$. Therefore, we have the following hamiltonian cycle in $\operatorname{Cay}\left(G / \mathbb{Z}_{2} ; S\right)$ :

$$
C=\left(c,\left(c^{\ell-2}, a, c^{-(\ell-2)}, b\right)^{p} \#, c^{-1},\left(a^{-1}, b^{-1}\right)^{p} \#\right) .
$$

Since $[a, b]$ projects trivially to $\mathbb{Z}_{2}$, Corollary 3.8 implies that $\ell$ is even, so, calculating modulo $\mathbb{Z}_{p}$, we have

$$
\begin{array}{rlrl}
\Pi C & =c\left(c^{\ell-2} a c^{-(\ell-2)} b\right)^{p} b^{-1} c^{-1}\left(a^{-1} b^{-1}\right)^{p} b & \\
& \equiv c(a b)^{p} b^{-1} c^{-1}\left(a^{-1} b^{-1}\right)^{p} b & & \left(\begin{array}{l}
\ell-2 \text { is even, so } c^{\ell-2} \\
\text { is central modulo } \mathbb{Z}_{p}
\end{array}\right) \\
& \equiv z^{2 p-1}(a b)^{p} b^{-1}\left(a^{-1} b^{-1}\right)^{p} b & & \left(\begin{array}{c}
\text { letting } z=[a, c]=[b, c] \text { be } \\
\text { the nontrivial element of } \mathbb{Z}_{2}
\end{array}\right) \\
& \equiv z & & \left(z^{2}=e \text { and }[a, b] \in \mathbb{Z}_{p}\right) .
\end{array}
$$

Since this generates $\mathbb{Z}_{2}$, the Factor Group Lemma (2.8) applies.

\section{Cases with $|\bar{a}|=2$ and $\# S \geq 4$}

Assumption 9.1. In this section, we assume

- $\# S \geq 4$, and

- $|\bar{s}|=2$, for all $s \in S$, such that $s$ does not centralize $G^{\prime}$. 
We also assume Case 4.1 does not apply. (So $|s|=2$.)

Furthermore, we assume $\bar{b} \notin\langle\bar{a}\rangle$ (otherwise, Case 4.1 applies). Then it is easy to see that we also have $\bar{a} \notin\langle\bar{b}\rangle$.

Outline. This final section of the proof is longer than the others, so here is an outline of the cases and subcases that it considers.

9.4: Assume no element of $S$ centralizes $G^{\prime}$.

9.4.1: Assume $\# S \geq 5$.

9.4.2: Assume $\# S=4$.

9.5: Assume there exists $s \in S$, such that $[a, s] \notin \mathbb{Z}_{p}$, and, in addition, either $s=b$, or b centralizes $G^{\prime}$, or $\mathbb{Z}_{p} \subseteq\langle S \backslash\{a\}\rangle^{\prime}$.

9.5.1: Assume $\mathbb{Z}_{p} \nsubseteq\langle S \backslash\{a\}\rangle^{\prime}$.

9.5.2: Assume $\mathbb{Z}_{p} \subseteq\langle S \backslash\{a\}\rangle^{\prime}$.

9.6: Assume b centralizes $G^{\prime}$.

9.6.1: Assume there exists $c \in S$, such that $[c, b] \notin \mathbb{Z}_{p}$.

9.6.2: Assume $[c, b] \in \mathbb{Z}_{p}$ for all $c \in S$.

9.7: Assume that none of the preceding cases apply.

Since Case 9.4 does not apply, some element $c$ of $S$ centralizes $G^{\prime}$.

9.7.1: Assume $\langle[s, c]\rangle \neq \mathbb{Z}_{2}$, for some $s \in S \backslash\{c\}$.

9.7.2: Assume $\langle[s, c]\rangle=\mathbb{Z}_{2}$, for all $s \in S \backslash\{c\}$.

Notation 9.2. Let $n=|\bar{b}|$ and $\ell=|\bar{G}:\langle\bar{a}, \bar{b}\rangle|=|\bar{G}| /(2 n)$.

Note 9.3. The irredundance of $S$ implies $S \backslash\{a, b\}$ is an irredundant generating set for $\bar{G} /\langle\bar{a}, \bar{b}\rangle$ (see Corollary 3.5), so $\ell \geq 4$.

Case 9.4. Assume no element of $S$ centralizes $G^{\prime}$.

Proof. From Assumption 9.1, we see that every element of $S$ inverts $G^{\prime}$ (and has order 2). We may assume no two elements of $S$ commute, for otherwise it is not difficult to see that Lemma 2.13 applies.

Let $c, d \in S \backslash\{a, b\}$, and let $\gamma=[a, b][a, c]$. We claim that we may assume $\gamma \notin \mathbb{Z}_{2}$, by permuting $b, c, d$. To this end, first note that if $\gamma \in \mathbb{Z}_{2}$, then $\mathbb{Z}_{p} \subseteq\langle[a, c]\rangle$, so there is no harm in putting $c$ into the role of $b$. Now, let us suppose $[a, b][a, c],[a, c][a, d]$, and $[a, d][a, b]$ are all in $\mathbb{Z}_{2}$. Then

$$
[a, b] \equiv[a, c]^{-1} \equiv[a, d] \equiv[a, b]^{-1} \quad\left(\bmod \mathbb{Z}_{2}\right),
$$

which contradicts the fact that $[a, b] \notin \mathbb{Z}_{2}$ (and $p$ is odd).

Let

$$
C=\left((c, a, c, b)^{2} \#, d\right)^{2},
$$

so $C$ is a hamiltonian cycle in $\operatorname{Cay}(\langle\bar{a}, \bar{b}, \bar{c}, \bar{d}\rangle ;\{a, b, c, d\})$ that contains the vertex-disjoint paths $[\bar{e}](c, a, c),[\overline{a b c}](a),[\overline{b d}](c, a, c)$, and $[\overline{a c d}](a)$. Applying Lemma 2.12 to the paths 
$[\bar{e}](c, a, c)$ and $[\overline{a b c}](a)$ (so $s=c, t=a, u=b$, and $h=b c$ ) will multiply the voltage by $\gamma$. Applying Lemma 2.12 to the other two paths $[\overline{b d}](c, a, c)$ and $[\overline{a c d}](a)$ (so $s=c$, $t=a, u=b$, and $h=c d$ ) will also multiply the voltage by $\gamma$ (because $b c$ and $c d$ both centralize $G^{\prime}$ ). Therefore, applying Lemma 2.12 twice yields a hamiltonian cycle $C^{\prime \prime}$, such that $(\Pi C)^{-1}\left(\Pi C^{\prime \prime}\right)=\gamma^{2}$, which is a generator of $\mathbb{Z}_{p}$.

Subcase 9.4.1. Assume $\# S \geq 5$. If there exist $s, t \in S$, such that $s \notin\{a, b, c\}$, and $[s, t] \notin \mathbb{Z}_{p}$, then the preceding paragraph implies that Lemma 3.15(2) applies.

Thus, we may assume that the preceding condition does not apply (for any legitimate choice of $a, b$, and $c$ ). Fix two elements $x, y \in S \backslash\{a, b, c\}$. The failure of the condition implies $[x, S] \subseteq \mathbb{Z}_{p}$. In particular, $[x, y]$ must be a generator of $\mathbb{Z}_{p}$ (because no two elements of $S$ commute), so we may let $\{x, y\}$ play the role of $\{a, b\}$. So we may let $\{x, y, b, c\}$ play the role of $\{a, b, c, d\}$. Then, since $a \notin\{x, y, b, c\}$, the failure of the condition implies $[a, S] \subseteq \mathbb{Z}_{p}$. Similarly, $[b, S]$ and $[c, S]$ are also in $\mathbb{Z}_{p}$. So $[s, t] \subseteq \mathbb{Z}_{p}$ for all $s, t \in S$. This contradicts the fact that $\langle[S, S]\rangle=G^{\prime} \nsubseteq \mathbb{Z}_{p}$.

Subcase 9.4.2. Assume $\# S=4$. For convenience, in this subcase (and only in this subcase), we drop our standing assumption that $\langle[a, b]\rangle$ contains $\mathbb{Z}_{p}$. Instead, choose $b, d \in$ $S$, such that $[b, d]$ projects nontrivially to $\mathbb{Z}_{2}$. A straightforward calculation (using the fact that $a, b, c$, and $d$ all invert $G^{\prime}$ ) shows that

$$
\Pi C=[c, d]^{4}[d, a]^{2}[d, b] .
$$

Since $[d, b]$ projects nontrivially to $\mathbb{Z}_{2}$, but $[c, d]^{4}$ and $[d, a]^{2}$ have even exponents, so they obviously do not, we see that $\mathbb{Z}_{2} \subseteq\langle\Pi C\rangle$. Therefore, we may assume $\Pi C \in \mathbb{Z}_{2}$, for otherwise the Factor Group Lemma (2.8) applies.

We may assume $\gamma \in \mathbb{Z}_{2}$, for otherwise applying Lemma 2.12 twice (as in the paragraph immediately before Subcase 9.4.1) yields a hamiltonian cycle whose voltage generates $G^{\prime}$, so the Factor Group Lemma (2.8) applies. By the definition of $\gamma$, this means $[a, b][a, c] \in$ $\mathbb{Z}_{2}$. And we may assume the same is true when $b$ and $d$ are interchanged, which means $[a, d][a, c] \in \mathbb{Z}_{2}$. So

$$
[a, b] \equiv[a, c]^{-1} \equiv[a, d] \quad\left(\bmod \mathbb{Z}_{2}\right) .
$$

By interchanging $a$ and $c$, we conclude that we may also assume

$$
[c, b] \equiv[c, a]^{-1} \equiv[c, d] \quad\left(\bmod \mathbb{Z}_{2}\right) .
$$

So

$$
[c, d] \equiv[c, a]^{-1}=[a, c] \equiv[a, d]^{-1}=[d, a] \quad\left(\bmod \mathbb{Z}_{2}\right)
$$

Therefore

$$
[d, a]^{6}[d, b]=[d, a]^{4}[d, a]^{2}[d, b] \equiv[c, d]^{4}[d, a]^{2}[d, b]=\Pi C \equiv 0 \quad\left(\bmod \mathbb{Z}_{2}\right) .
$$

If $p \neq 3$, then, since we may assume the same is true when we interchange $a$ and $c$, we conclude that $[d, c] \equiv[d, a]\left(\bmod \mathbb{Z}_{2}\right)$. Since we also have $[c, d] \equiv[d, a]\left(\bmod \mathbb{Z}_{2}\right)$, we conclude that $[c, d]$ and $[a, d]$ are in $\mathbb{Z}_{2}$. This implies $[b, d] \notin \mathbb{Z}_{2}$ (since $d$ does not centralize $\mathbb{Z}_{p}$, and is therefore not in the center of $G / \mathbb{Z}_{2}$ ), so

$$
\Pi C=[c, d]^{4}[d, a]^{2}[d, b] \equiv e^{4} e^{2}[d, b]=[d, b] \not \equiv 0 \quad\left(\bmod \mathbb{Z}_{2}\right) .
$$

This contradicts the fact that $\Pi C \in \mathbb{Z}_{2}$. 
We now assume $p=3$. Then the equation $[d, a]^{6}[d, b] \equiv 0\left(\bmod \mathbb{Z}_{2}\right)$ implies $[d, b] \in$ $\mathbb{Z}_{2}$. This conclusion came from assuming only that $[d, b] \notin \mathbb{Z}_{p}$. Therefore, for all $s, t \in S$, the commutator $[s, t]$ must be in either $\mathbb{Z}_{2}$ or $\mathbb{Z}_{p}$. However,

$$
[a, b] \equiv[c, a] \equiv[a, d] \equiv[b, c] \equiv[d, c] \quad\left(\bmod \mathbb{Z}_{2}\right),
$$

and $[a, b] \notin \mathbb{Z}_{2}$. Therefore, we conclude all five of these other commutators are in $\mathbb{Z}_{p}$. (Therefore, the stated congruences between these commutators are actually equalities.)

Now, interchanging $a \leftrightarrow b$ and $c \leftrightarrow d$ in $C$ yields a hamiltonian cycle $C^{*}$, such that

$$
\Pi C^{*}=[d, c]^{4}[c, b]^{2}[c, a]=[d, c][b, c][c, a]=[c, a]^{3}=e
$$

(because $p=3$ ). Let $\gamma^{*}=[b, a][b, d]$, so $\gamma^{*}$ is obtained from $\gamma=[a, b][a, c]$ by interchanging $a \leftrightarrow b$ and $c \leftrightarrow d$. Then, since applying Lemma 2.12 to $C$ can multiply the voltage by $\gamma=[a, b][a, c]$, we know that applying Lemma 2.12 to $C^{*}$ can multiply the voltage by $\gamma^{*}$, which generates $G^{\prime}$. So the Factor Group Lemma (2.8) applies.

Case 9.5. Assume there exists $s \in S$, such that $[a, s] \notin \mathbb{Z}_{p}$, and:

$$
\text { either } s=b \text {, or b centralizes } G^{\prime} \text {, or } \mathbb{Z}_{p} \subseteq\langle S \backslash\{a\}\rangle^{\prime} \text {. }
$$

Proof. Let $S_{0}=S \backslash\{a\}$. Note that the irredundance of $S$ implies $a \notin\left\langle S_{0}\right\rangle \mathbb{Z}_{2}$ (see Lemma 3.4).

Subcase 9.5.1. Assume $\mathbb{Z}_{p} \nsubseteq\left\langle S_{0}\right\rangle^{\prime}$. If $[a, b] \notin \mathbb{Z}_{p}$, we assume that $s=b$. Let

$$
g= \begin{cases}s & \text { if }[s, a] \notin \mathbb{Z}_{2}, \\ s b^{2} & \text { if }[s, a] \in \mathbb{Z}_{2} .\end{cases}
$$

Note that $\langle[g, a]\rangle=G^{\prime}$.

Let $H^{*}=\left\langle S_{0}\right\rangle \mathbb{Z}_{2} / \mathbb{Z}_{2}$. From the assumption of this subcase, we know that $H^{*}$ is abelian. Therefore, Corollary 2.11 provides a hamiltonian path $L=\left(s_{i}\right)_{i=1}^{r}$ in $\operatorname{Cay}\left(\overline{H^{*}} ; S_{0}\right)$, such that $s_{1} s_{2} \cdots s_{r} \in g \mathbb{Z}_{2}$. Then $\left(L^{-1}, a, L, a\right)$ is a hamiltonian cycle in $\operatorname{Cay}(\bar{G} ; S)$, and

$$
\Pi C=\left[s_{1} s_{2} \cdots s_{r}, a\right] \in\left[g \mathbb{Z}_{2}, a\right]=\{[g, a]\}
$$

(since $\mathbb{Z}_{2}$ is in the center of $G$ ). This voltage generates $G^{\prime}$, so the Factor Group Lemma (2.8) applies.

Subcase 9.5.2. Assume $\mathbb{Z}_{p} \subseteq\left\langle S_{0}\right\rangle^{\prime}$. Suppose $w, x, y \in S^{ \pm 1} \backslash\{a\}$, such that

$$
\langle\bar{w}\rangle \subsetneq\langle\bar{w}, \bar{x}\rangle \subsetneq\langle\bar{w}, \bar{x}, \bar{y}\rangle
$$

It is easy to construct a hamiltonian cycle $C_{0}$ in $\operatorname{Cay}\left(\left\langle\overline{S_{0}}\right\rangle ; S_{0}\right)$, such that $C_{0}$ contains the oriented paths $\left[\overline{h w^{-1} y^{-1}}\right]\left(w, x, w^{-1}\right)$ and $[\overline{h x}]\left(x^{-1}\right)$, for some $h \in G$. Furthermore, if

$$
\text { either } x \notin\left\{s^{ \pm 1}\right\} \text { or }|\bar{G}|>16
$$

then, for some $\epsilon \in\{ \pm 1\}$, it is not difficult to arrange that the hamiltonian cycle $C_{0}$ contains the oriented edge $\left[\overline{s^{\epsilon}}\right]\left(s^{-\epsilon}\right)$, and that this edge is not in either of the above-mentioned paths. 
Applying Lemma 2.12 to the first two paths (so $s=w, t=x$, and $u=y$ ) yields a hamiltonian cycle $C_{1}$, such that $\left(\Pi C_{0}\right)^{-1}\left(\Pi C_{1}\right)$ is conjugate to $\left[x^{-1}, y\right]\left[w, x^{-1}\right]^{y}$. $\operatorname{Re}-$ moving the edge $\left[s^{\epsilon}\right]\left(s^{-\epsilon}\right)$ yields hamiltonian paths $C_{0} \#$ and $C_{1} \#$ from $\bar{e}$ to $\bar{s}^{\epsilon}$.

From Lemma 3.4 and the assumption of this subcase, we see that $\left\langle\overline{S_{0}}\right\rangle \neq \bar{G}$. So

$$
C_{0}^{+}=\left(C_{0} \#, a,\left(C_{0} \#\right)^{-1}, a\right) \text { and } C_{1}^{+}=\left(C_{1} \#, a,\left(C_{1} \#\right)^{-1}, a\right)
$$

are hamiltonian cycles in $\operatorname{Cay}(\bar{G} ; S)$. For $k=0,1$, we have

$$
\Pi C_{k}^{+}=\left[\left(\left(\Pi C_{k}\right) s^{\epsilon}\right)^{-1}, a\right]
$$

Since $\Pi C_{k} \in G^{\prime}$, and $G^{\prime}$ is central modulo $\mathbb{Z}_{p}$ (and from the choice of $s$ ), we have

$$
\Pi C_{k}^{+} \equiv\left[s^{\epsilon}, a\right] \not \equiv e \quad\left(\bmod \mathbb{Z}_{p}\right) .
$$

Furthermore, if $\left[x^{-1}, y\right]\left[w, x^{-1}\right]^{y}$ projects nontrivially to $\mathbb{Z}_{p}$, then $\left(\Pi C_{0}^{+}\right)^{-1}\left(\Pi C_{1}^{+}\right)$ does not centralize $a$ modulo $\mathbb{Z}_{2}$, so $\Pi C_{0}^{+}$and $\Pi C_{1}^{+}$are not both in $\mathbb{Z}_{2}$. This implies that $\Pi C_{k}^{+}$generates $G^{\prime}$ for some $k$, so the Factor Group Lemma (2.8) applies. Therefore (after replacing $x^{-1}$ with $x$ for simplicity), we may assume

$$
[w, x]^{y}[x, y] \in \mathbb{Z}_{2} \text { for all } w, x, y \in S^{ \pm 1} \backslash\{a\} \text { that satisfy (9.5A) and (9.5B). }
$$

We will show that this leads to a contradiction.

Assume, for the moment, that $b$ centralizes $G^{\prime}$. Then $n=|\bar{b}|>2$ (because Corollary 3.7 implies that $|b| \neq 2$ ), so $|\bar{G}|=2 n \ell>2 \cdot 2 \cdot 4=16$. Therefore (9.5B) is automatically satisfied. Let $x, y \in S_{0} \backslash\{b\}$, such that $x \neq y$. We see from Note 9.3 that (9.5A) is satisfied for $w=b^{ \pm 1}$, so (9.5C) tells us

$$
[b, x]^{y}[x, y] \text { and }\left[b^{-1}, x\right]^{y}[x, y] \text { are both in } \mathbb{Z}_{2} \text {. }
$$

However, we also know that $\left[b^{-1}, x\right]=[b, x]^{-1}$ (because we are assuming in this paragraph that $b$ centralizes $G^{\prime}$ ). Therefore

$$
[b, x]^{y} \equiv[x, y]^{-1} \equiv\left[b^{-1}, x\right]^{y}=\left([b, x]^{-1}\right)^{y} \quad\left(\bmod \mathbb{Z}_{2}\right),
$$

so $[b, x] \in \mathbb{Z}_{2}$ (for all $x \in S_{0}$ ). Then, since $[b, x]^{y}[x, y] \in \mathbb{Z}_{2}$, we conclude that $[x, y] \in \mathbb{Z}_{2}$, for all $x, y \in S_{0}$. This contradicts the assumption of this subcase.

Now assume $b$ does not centralize $G^{\prime}$. We may assume Case 9.4 does not apply, so $G^{\prime}$ is centralized by some $t \in S$ (and $t \neq b$ ). Let $w, x \in S_{0} \backslash\{t\}$ with $w \neq x$. Combining the irredundance of $S$ with the fact that $t \neq b$ implies that $(9.5 \mathrm{~A})$ is satisfied for $y=t^{ \pm 1}$ (unless $\bar{w}=\bar{x}$, when Case 4.1 applies). We may assume $x \neq s$ (by interchanging $w$ and $x$, if necessary), so (9.5B) is satisfied. Then (9.5C) tells us

$$
[w, x]^{t}[x, t] \text { and }[w, x]^{t^{-1}}\left[x, t^{-1}\right] \text { are both in } \mathbb{Z}_{2} .
$$

Since $t$ centralizes $G^{\prime}$, this implies $[x, t] \equiv\left[x, t^{-1}\right]=[x, t]^{-1}\left(\bmod \mathbb{Z}_{2}\right)$, so $[x, t] \in \mathbb{Z}_{2}$ (for all $x \in S_{0}$ ). Since $[w, x]^{t}[x, t] \in \mathbb{Z}_{2}$, this implies $[w, x] \in \mathbb{Z}_{2}$ (for all $w, x \in S_{0}$ ). This contradicts the assumption of this subcase.

Case 9.6. Assume b centralizes $G^{\prime}$. 
Proof. We consider two subcases.

Subcase 9.6.1. Assume there exists $c \in S$, such that $[c, b] \notin \mathbb{Z}_{p}$. We use some of the arguments of Case 8.5. We may assume $[a, s] \in \mathbb{Z}_{p}$ for all $s \in S$. (Otherwise, Case 9.5 applies, because $b$ centralizes $G^{\prime}$.) Therefore $c \neq a$. Let $L=\left(s_{i}\right)_{i=1}^{r}$ be a hamiltonian path from $\bar{e}$ to $\bar{b}$ in $\operatorname{Cay}(\bar{G} /\langle\bar{a}\rangle ; S \backslash\{a\})$, such that $s_{1}=c=s_{r}^{-1}$, and $L$ contains a path of the form $\left[\overline{g c^{\epsilon}}\right]\left(c^{-\epsilon}, b^{\delta}, c^{\epsilon}\right)$ (for some $\delta, \epsilon \in\{ \pm 1\}$ ) that is vertex-disjoint from $\{\bar{e}, \bar{c}, \bar{b}, \overline{b c}\}$. Now let $C=\left(L, a, L^{-1}, a\right)$. Then $C$ contains vertex-disjoint paths of the form

$$
[\bar{b}](a), \quad[\overline{c a}]\left(c^{-1}, a, c\right), \quad\left[\overline{g c^{\epsilon}}\right]\left(c^{-\epsilon}, b^{\delta}, c^{\epsilon}\right), \quad \text { and } \quad\left[\overline{g a b^{\delta}}\right]\left(b^{-\delta}\right) .
$$

- Applying Lemma 2.12 to $[\bar{b}](a)$ and $[\overline{c a}]\left(c^{-1}, a, c\right)$ (so $s=c^{-1}, t=a, u=b$, and $h=a b)$ will be called the " $a$-transform." It multiplies the voltage by

$$
\gamma_{a}=[b, a]\left[a, c^{-1}\right]
$$

- Applying Lemma 2.12 to $\left[\overline{g c^{\epsilon}}\right]\left(c^{-\epsilon}, b^{\delta}, c^{\epsilon}\right)$ and $\left[\overline{g a b^{\delta}}\right]\left(b^{-\delta}\right)$ (so $s=c^{-\epsilon}, t=b^{\delta}$, $u=a$, and $h=g a$ ) will be called the " $b$-transform." It multiplies the voltage by a conjugate of

$$
\gamma_{b}=[a, b]\left[c^{-\epsilon}, b\right]
$$

Since $[a, b],[a, c] \in \mathbb{Z}_{p}$ and $[b, c] \notin \mathbb{Z}_{p}$ we know $\gamma_{a} \in \mathbb{Z}_{p}$ and $\gamma_{b} \notin \mathbb{Z}_{p}$. Also, we may also assume $\gamma_{a}$ is nontrivial (by replacing $b$ with $b^{-1}$ if necessary). Therefore, the argument of Subcase 8.5.1 applies. Namely, choose $C^{\prime}$ to be either $C$ or the $b$-transform of $C$, such that $\Pi C^{\prime}$ projects nontrivially to $\mathbb{Z}_{2}$. Then choose $C^{\prime \prime}$ to be either $C^{\prime}$ or the $a$-transform of $C^{\prime}$, such that $\Pi C^{\prime \prime}$ generates $G^{\prime}$, so the Factor Group Lemma (2.8) applies.

Subcase 9.6.2. Assume $[c, b] \in \mathbb{Z}_{p}$ for all $c \in S$. Choose $c, d \in S$, such that $[c, d] \notin \mathbb{Z}_{p}$. Assuming that Case 9.5 and Subcase 9.6.1 do not apply, we have

$$
[s, t] \in \mathbb{Z}_{p} \text { for all } s \in\{a, b\} \text { and } t \in S .
$$

Therefore, $c, d \notin\{a, b\}$, and the element $\gamma=[a, b]\left[d^{-1}, a\right]$ is in $\mathbb{Z}_{p}$, and we may assume (by replacing $b$ with its inverse, if necessary) that $\gamma$ generates $\mathbb{Z}_{p}$.

Let $S_{0}=\{a, b, d\}$, and choose a hamiltonian cycle $C_{0}$ in Cay $\left(\left\langle\overline{S_{0}}\right\rangle ; S_{0}\right)$ that contains the oriented paths $[\bar{d}]\left(d^{-1}, a, d\right)$ and $[\overline{a b}](a)$, and has at least two edges labelled $x^{ \pm 1}$, for every $x \in S_{0}$. Lemma 2.12 (with $s=d^{-1}, t=a, u=b$, and $h=b$ ) provides a hamiltonian cycle $C_{1}$, such that $\left(\Pi C_{0}\right)^{-1}\left(\Pi C_{1}\right)$ is conjugate to $\gamma$, and therefore generates $\mathbb{Z}_{p}$. Furthermore, $C_{1}$ contains all of the oriented edges of $C_{0}$ that are not in these two above-mentioned paths, so Lemma 3.15(2) applies (with $g=b$ and $t=d$ ).

Case 9.7. Assume that none of the preceding cases apply.

Proof. This implies that:

\#1. $[a, b] \in \mathbb{Z}_{p}$. (Otherwise, Case 9.5 applies.)

\#2. If $s \in S$, and there exists $t \in S$, such that $t$ inverts $G^{\prime}$ and $\mathbb{Z}_{p} \subseteq\langle[s, t]\rangle$, then $s$ inverts $G^{\prime}$. (If $s$ does not invert $G^{\prime}$, then we see from Assumption 9.1 that $s$ centralizes $G^{\prime}$, so Case 9.6 applies with $s$ and $t$ in the roles of $b$ and $a$, respectively.) 
\#3. There exists $c \in S$, such that c centralizes $G^{\prime}$. (Otherwise, Case 9.4 applies.) From (\#2), we know $[a, c] \in \mathbb{Z}_{2}$.

Subcase 9.7.1. Assume $\langle[s, c]\rangle \neq \mathbb{Z}_{2}$, for some $s \in S \backslash\{c\}$. Suppose, for the moment, that $s$ centralizes $G^{\prime}$. Then Lemma 3.6 implies $[a,[s, c]]=[[a, s],[a, c]]=e$ (because $G^{\prime}$ is abelian), so $[s, c]$ projects trivially to $\mathbb{Z}_{p}$. Since $\langle[s, c]\rangle \neq \mathbb{Z}_{2}$, we conclude from this that $[s, c]=e$, so Lemma 2.16 applies.

We may now assume $s$ does not centralize $G^{\prime}$, so there is no harm in assuming that $s=$ $a$. Since (\#2) implies that $[a, c] \in \mathbb{Z}_{2}$, we see that $[a, c]$ must be trivial. Let $H=\langle S \backslash\{c\}\rangle$. We may assume $\mathbb{Z}_{2} \nsubseteq H$, for otherwise $H \triangleleft G$, so Lemma 2.13 applies with $s=c$ and $t=a$. Therefore, $[x, y] \in \mathbb{Z}_{p}$ for all $x, y \in S \backslash\{c\}$, but there is some $d \in S \backslash\{c\}$, such that $[c, d]$ projects nontrivially to $\mathbb{Z}_{2}$.

Similarly, we may assume $\mathbb{Z}_{p} \nsubseteq\langle S \backslash\{a\}\rangle$, for otherwise we have $\langle S \backslash\{a\}\rangle \triangleleft G$, so Lemma 2.13 applies with $s=a$ and $t=c$. This means $[x, y] \in \mathbb{Z}_{2}$ for all $x, y \in S \backslash\{a\}$. In particular, since $b$ and $d$ are in both $S \backslash\{a\}$ and $S \backslash\{c\}$, we must have $[b, d] \in \mathbb{Z}_{2} \cap \mathbb{Z}_{p}=$ $\{e\}$.

Choose a hamiltonian cycle $C_{0}$ in $\operatorname{Cay}(\bar{H} ; S \backslash\{c\})$ that contains the oriented paths $[\bar{d}]\left(d^{-1}, b, d\right)$ and $[\overline{a b}](b)$. If we apply Lemma 2.12 to these paths (so $s=d^{-1}, t=b$, $u=a$, and $h=a$ ), then the voltage is multiplied by a conjugate of $[b, a]\left[b, d^{-1}\right]$, which is a generator of $\mathbb{Z}_{p}$ (since $[a, b]$ generates $\mathbb{Z}_{p}$ and $[b, d]$ is trivial). Therefore, Lemma 3.15(1) applies with $s=t=d$ and $u=a$.

Subcase 9.7.2. Assume $\langle[s, c]\rangle=\mathbb{Z}_{2}$, for all $s \in S \backslash\{c\}$. For convenience, let $\widehat{G}=G / \mathbb{Z}_{2}$ and $\widehat{H}=\langle\widehat{S} \backslash\{\widehat{c}\}\rangle$. Then $\left|\widehat{H}^{\prime}\right|=p$ is prime, so Theorem 1.1 provides a hamiltonian path $L$ in $\operatorname{Cay}(\widehat{H} ; S \backslash\{c\})$. Since $\widehat{c}$ is central in $\widehat{G}$, there is a spanning subgraph of $\operatorname{Cay}(\widehat{G} ; S)$ that is isomorphic to the Cartesian product $L \square\left(\widehat{c}^{\ell-1}\right)$, where $\ell=|\bar{G}:\langle\overline{S \backslash\{c\}}\rangle|$. Since $|\widehat{G}|$ is even, it is easy to find a hamiltonian cycle $C$ in $L \square\left(\widehat{c}^{\ell-1}\right)$ (see Lemma 2.10), and this yields a hamiltonian cycle $\widehat{C}$ in $\operatorname{Cay}(\widehat{G} ; S)$.

To complete the proof, we carry out a straightforward (and well-known) calculation to verify that $\Pi \widehat{C}$ is nontrivial, so the Factor Group Lemma (2.8) applies.

If we view the Cartesian product $L \square\left(\widehat{c}^{\ell-1}\right)$ as a grid of squares, then the interior of the hamiltonian cycle $C$ is a union of squares of the grid. Graph theoretically, this means $C$ is the connected sum of some number $N$ of digons of the form $[g]\left(t, t^{-1}\right)$ (where $t \in S^{ \pm 1}$ ). Note that if $\mathcal{C}$ is an $r$-cycle (with $r \geq 2)$, then $\mathcal{C} \#_{t}^{s}\left(t, t^{-1}\right)$ is an $(r+2)$-cycle. Therefore, since the length of $C$ is $|\widehat{G}|$, we have $2 N=|\widehat{G}| \equiv 0(\bmod 4)$, so $N$ is even.

Now, each 4-cycle in $L \square\left(\hat{c}^{\ell-1}\right)$ is of the form $[\widehat{g}]\left(s^{-1}, t^{-1}, s, t\right)$, where one of $s$ and $t$ is in $\left\{c^{ \pm 1}\right\}$, and the other is in $S^{ \pm 1} \backslash\left\{c^{ \pm 1}\right\}$. This means that in any connected sum $\mathcal{C} \#_{t}^{s}[g]\left(t, t^{-1}\right)$, one of $s$ and $t$ is in $\left\{c^{ \pm 1}\right\}$, and the other is in $S^{ \pm 1} \backslash\left\{c^{ \pm 1}\right\}$. By the assumption of this subcase, we conclude that $[s, t]=z$, where $z$ is the generator of $\mathbb{Z}_{2}$. Therefore

$$
\begin{array}{rlrl}
\Pi C & =\Pi\left(\left[\widehat{g_{1}}\right]\left(t_{1}, t_{1}^{-1}\right) \#_{t_{2}}^{s_{2}}\left[\widehat{g_{2}}\right]\left(t_{2}, t_{2}^{-1}\right) \#_{t_{3}}^{s_{3}} \cdots \#_{t_{N}}^{s_{N}}\left[\widehat{g_{N}}\right]\left(t_{N}, t_{N}^{-1}\right)\right) \\
& \equiv \prod_{i=2}^{N}\left[s_{i}, t_{i}\right] & & \left(\text { Corollary } 3.14 \text { and } \Pi\left(t, t^{-1}\right)=e\right) \\
& =z^{N-1} & & (N-1 \text { is odd }) .
\end{array}
$$




\section{References}

[1] K. Bannai, Hamiltonian cycles in generalized Petersen graphs, J. Comb. Theory Ser. B 24 (1978), 181-188, doi:10.1016/0095-8956(78)90019-9.

[2] C. C. Chen and N. F. Quimpo, On strongly Hamiltonian abelian group graphs, in: K. L. McAvaney (ed.), Combinatorial Mathematics VIII, Springer, Berlin, volume 884 of Lecture Notes in Mathematics, pp. 23-34, 1981, doi:10.1007/bfb0091805, proceedings of the Eighth Australian Conference on Combinatorial Mathematics Held at Deakin University, Geelong, August 25 29, 1980.

[3] S. J. Curran, D. Witte Morris and J. Morris, Cayley graphs of order $16 p$ are hamiltonian, Ars Math. Contemp. 5 (2012), 185-211, http: / / amc-journal. eu/index . php/amc/ article/view/207.

[4] E. Durnberger, Connected Cayley graphs of semi-direct products of cyclic groups of prime order by abelian groups are Hamiltonian, Discrete Math. 46 (1983), 55-68, doi:10.1016/ $0012-365 \times(83) 90270-4$.

[5] E. Durnberger, Every connected Cayley graph of a group with prime order commutator group has a Hamilton cycle, in: B. R. Alspach and C. D. Godsil (eds.), Cycles in Graphs, NorthHolland, Amsterdam, volume 115 of North-Holland Mathematics Studies, pp. 75-80, 1985, doi:10.1016/s0304-0208(08)72997-9, papers from the workshop held at Simon Fraser University, Burnaby, B.C., July 5 - August 20, 1982.

[6] E. Ghaderpour and D. Witte Morris, Cayley graphs of order $27 p$ are hamiltonian, Int. J. Comb. 2011 (2011), Article ID 206930 (16 pages), doi:10.1155/2011/206930.

[7] E. Ghaderpour and D. Witte Morris, Cayley graphs of order 30p are hamiltonian, Discrete Math. 312 (2012), 3614-3625, doi:10.1016/j.disc.2012.08.017.

[8] E. Ghaderpour and D. Witte Morris, Cayley graphs on nilpotent groups with cyclic commutator subgroup are hamiltonian, Ars Math. Contemp. 7 (2014), 55-72, http: / / amc-journal. eu/index.php/amc/article/view/280.

[9] C. Godsil and G. Royle, Algebraic Graph Theory, volume 207 of Graduate Texts in Mathematics, Springer-Verlag, New York, 2001, doi:10.1007/978-1-4613-0163-9.

[10] J. L. Gross and T. W. Tucker, Topological Graph Theory, Wiley-Interscience Series in Discrete Mathematics and Optimization, John Wiley \& Sons, Inc., New York, 1987.

[11] K. Keating and D. Witte, On Hamilton cycles in Cayley graphs in groups with cyclic commutator subgroup, in: B. R. Alspach and C. D. Godsil (eds.), Cycles in Graphs, NorthHolland, Amsterdam, volume 115 of North-Holland Mathematics Studies, pp. 89-102, 1985, doi:10.1016/S0304-0208(08)72999-2, papers from the workshop held at Simon Fraser University, Burnaby, B.C., July 5 - August 20, 1982.

[12] K. Kutnar, D. Marušič, D. Witte Morris, J. Morris and P. Šparl, Hamiltonian cycles in Cayley graphs whose order has few prime factors, Ars Math. Contemp. 5 (2012), 27-71, http: // amc-journal.eu/index.php/amc/article/view/177.

[13] D. Marušič, Hamiltonian circuits in Cayley graphs, Discrete Math. 46 (1983), 49-54, doi: 10.1016/0012-365x(83)90269-8.

[14] E. Schenkman, Group Theory, Robert E. Krieger Publishing Co., Huntington, New York, 1975, corrected reprint of the 1965 edition.

[15] D. Witte and J. A. Gallian, A survey: Hamiltonian cycles in Cayley graphs, Discrete Math. 51 (1984), 293-304, doi:10.1016/0012-365x(84)90010-4. 
D. W. Morris: Cayley graphs on groups with commutator subgroup of order $2 p$ are hamiltonian 31

[16] D. Witte Morris, Odd-order Cayley graphs with commutator subgroup of order $p q$ are hamiltonian, Ars Math. Contemp. 8 (2015), 1-28, http: / / amc-journal.eu/index.php/ amc/article/view/330. 